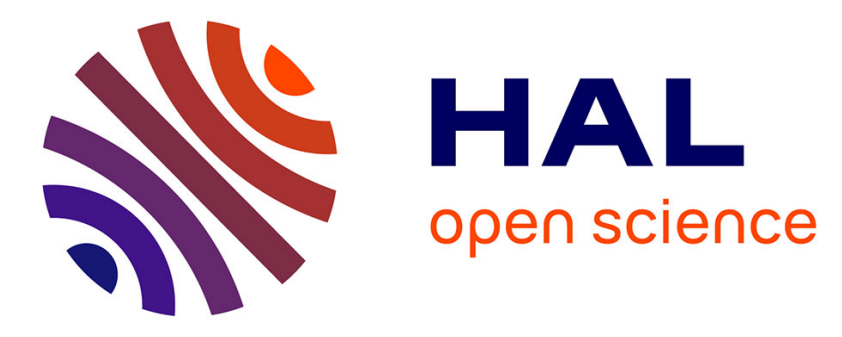

\title{
Complementary signal structure proposals for future airborne SBAS L5 signal and other non-airborne applications
}

Axel Javier Garcia Peña, Christophe Macabiau, Mikaël Mabilleau, Catalina Rodriguez, Daniel Brocard

\section{To cite this version:}

Axel Javier Garcia Peña, Christophe Macabiau, Mikaël Mabilleau, Catalina Rodriguez, Daniel Brocard. Complementary signal structure proposals for future airborne SBAS L5 signal and other nonairborne applications. IEEE/ION PLANS 2014, Position Location and Navigation Symposium, May 2014, Monterey, United States. pp.334-350, 10.1109/PLANS.2014.6851391 . hal-00989702

\section{HAL Id: hal-00989702 \\ https://hal-enac.archives-ouvertes.fr/hal-00989702}

Submitted on 1 Jul 2014

HAL is a multi-disciplinary open access archive for the deposit and dissemination of scientific research documents, whether they are published or not. The documents may come from teaching and research institutions in France or abroad, or from public or private research centers.
L'archive ouverte pluridisciplinaire HAL, est destinée au dépôt et à la diffusion de documents scientifiques de niveau recherche, publiés ou non, émanant des établissements d'enseignement et de recherche français ou étrangers, des laboratoires publics ou privés. 


\section{Complementary signal structure proposals for future airborne SBAS L5 signal and other non- airborne applications}

\author{
A. Garcia-Pena, C. Macabiau \\ ENAC \\ garcia-pena@recherche.enac.fr; \\ macabiau@recherche.enac.fr \\ C. Rodriguez, D. Brocard \\ CNES \\ catalina.rodriguez@cnes.fr \\ daniel.brocard@cnes.fr
}

\author{
M. Mabilleau \\ Egis Avia \\ Mikael.Mabilleau@egis.fr
}

\begin{abstract}
This paper studies complementary signal structures to the SBAS L5 SIS ICD definition which increases its original bit rate without degrading or even improving the SBAS L5 SIS ICD signal key performances. The proposition of this paper is based on modifying only certain characteristics of the current SBAS L5 SIS ICD signal structure and on keeping the signal characteristics which allow a perfect compatibility with SBAS L5 SIS ICD message content: symbol rate, bit information rate $(250 \mathrm{bps})$, basic information units of 250 bits and capability of interrupting the nominal message at each second in order to transmit alarm messages.

The main signal design modifications are first the introduction of linear block channel codes, instead of a convolutional code, in order to reduce header and CRC bits and in order to decrease the signal demodulation threshold. Second, new signal components, such as the synchronization component or a second data component, are introduced allowing an easier synchronization and increasing the effective bit rate. Smart combinations of the two data components are suggested to not degrade the final demodulation threshold. Finally, the selective introduction of a Code Shift Keying (CSK) modulation is also proposed to further increase the bit rate.
\end{abstract}

Demodulation, carrier phase tracking and secondary PRN code acquisition performances are explained and analyzed for the complementary proposed signal structures.

Keywords-SBAS; bit rate; carrier phase tracking; CSK; cycle slip rate; data component; demodulation performance; linear block channel codes; signal structure, synchronization; WER

\section{INTRODUCTION}

The SBAS L1 signal was designed in the late 90s in order to provide ranging plus integrity and correction data from Geostationary satellites for civil aviation applications to the only available core GNSS constellation and signal at that time, GPS L1 C/A. The SBAS L1 signal design was specifically optimized for a well determined set of system characteristics of that epoch: GPS constellation number of satellites, satellites atomic clock offset and drift characteristics at that epoch, GPS L1 C/A signal selectivity availability (SA), targeted approach procedures with vertical guidance (APV I), etc [1][2]. Therefore, the signal characteristics such as message content, implemented channel code, number of channel components, useful bit rate, etc., were selected accordingly to the fulfillment of these needs [1][2].

Nowadays, the majority of these system characteristics/ targets have changed or evolved: SA has been disabled, new atomic clocks are much more stable than the old ones, and approaches with vertical guidance with a decision height down to $200 \mathrm{ft}$ (LPV 200) are targeted. Moreover, a new GNSS signal in the L5 band, GPS L5, has been designed specifically for civil aviation requirements. Finally, more core GNSS constellations (with its own civil aviation oriented signals such as GALILEO E5) are being and will be deployed in the upcoming years.

Therefore, the design of a new SBAS signal, called L5, which includes the future multi-constellation horizon, the advantages provided by the new L5 civil oriented signals exploitation and which takes into account all the system characteristics/targets evolutions is mandatory.

Current proposals for the SBAS L5 signal under elaboration at Interoperability Working Group (IWG) are focused on designing a new L5 GEO signal by changing only the message structure and content from L1 GEO signal in order to have a SBAS signal generation transition as smooth as possible [3][4][5]. SBAS Interoperability Working Group (IWG) is the forum for SBAS service providers to assure common understanding and implementation of the ICAO SARPs. It allows coordinated development of interchangeable avionics technology designed to easily transition from one SBAS region to another.

The current IWG SBAS L5 SIS ICD definition, in addition to correcting weak points of the original SBAS L1 signal (poor quantization for the new stable atomic clocks and precise orbits, corrections spread on too many messages), have as main objective to free signal bandwidth in order to be able to accommodate up to 4 different core GNSS constellations around 90 satellites. 
However, the amount of signal bandwidth which can be freed is limited by the original signal design and thus the SBAS system improvement remains de facto limited. Therefore, although ongoing work of optimization of SBAS L5 ICD at IWG is expected to cover the needs of EGNOS V3 missions (incl. LPV200 and Cat I autoland) [6], the operation targets could become more ambitious and new services could also be aimed at (e.g. non-aero) if a larger bandwidth was available.

The aim of this paper is thus to study a complementary SBAS L5 signal structure proposal which provides enough bandwidth to accommodate up to 4 constellations (with about the same demodulation performance as for SBAS L1) and which even saves signal bandwidth for the transmission of enhanced aviation positioning services and other non-aviation applications (such as railway or maritime). It is important to remark that this study has to be seen as a possible evolution/complement (modification of the current signal design) of the current SBAS L5 SIS ICD definition (which only modifies the message content and structure).

In this article, first the motivations of the work are presented and second, the requirements derived from these motivations are defined. Third, the advanced signal structure techniques employed in this work to meet the requirements are described and fourth, their performances are presented. Sixth, the signal structure candidates are identified and their performances summarized. Finally, positioning services (airborne and non-airborne) are identified for each one of the candidates.

\section{Motivation}

The motivation of this paper consists in presenting several candidates and ideas for a new SBAS L1/L5 signal design which pursue 3 main goals:

1) Increase of the useful information bit rate $\left(R_{d}\right)$. The useful information bit rate or data rate, $R_{d}$, is the number of data information bits transmitted per second, and $R_{d}$ can be derived from the bit information rate, $R_{b}$, which is the number of information bits transmitted per second. In fact, the information bits of a message are equal to the addition of the data information bits, of the header bits (synchronization, message ID bits) and of the CRC bits; therefore, $R_{d}$ and $R_{b}$ can be related as:

$$
R_{d}=R_{b} \cdot \frac{\text { Data Bits }}{\text { Data Bits }+ \text { Header Bits }+ \text { CRC Bits }}
$$

In consequence, the increase of the data rate, $R_{d}$, can be obtained by either increasing $R_{b}$ and/or by decreasing the number of header and $\mathrm{CRC}$ bits with respect to the data bits.

2) Compatibility with the current SBAS L5 SIS ICD signal under elaboration at IWG [3][4][5].

3) Preservation (or even improving if possible) of the SBAS L5 SIS ICD definition signal performance (mainly the demodulation performance).

First, the increase of the data rate is the main goal being pursued in this work since a larger available $R_{d}$ could allow the SBAS system to target more ambitious operations, especially in the case where 4 constellations are simultaneously monitored. For instance, the broadcast of more parameters to users could allow to still enhance the aviation positioning services and to target CAT I autoland or even CAT II approaches with more performance margins:

- Bias terms of residual errors after application of SBAS SV corrections (clock, clock rate, ephemerides) in addition to variances

- More satellite DFREI parameters in MT6 [3] (further increase of the exclusion probability of side impacts on performance when 91 satellites are monitored)

- Degradation parameters per individual SV or per block of SV instead of per constellation (different satellites can have different integrity degradation performances)

- Information on SV signal distortions

- Ionospheric corrections and alarms for L5-only in order to allow improved operational capability in environments where the L1 frequency is experiencing interferences

Additionally, integrity information adapted to other user communities than aviation, such as the railway or the maritime communities could also be broadcasted, and other additional services could be targeted:

- GNSS authentication parameters

- Flexible integrity parameters for railway or maritime communities (non as stringent as for aero integrity risk, so that availability can be improved).

\section{- $\quad$ ARAIM parameters}

Second, the proposed signal structures should be $100 \%$ compatible with the current SBAS L5 SIS ICD signal under elaboration at IWG [3][4][5] (main characteristics presented in the next section). In fact, the definition of a new signal structure should be seen as a last (and optional) step of the SBAS L5 signal definition. First and foremost, the improvement of the navigation message with respect to SBAS L1 navigation message is carried out in order to correct weak points and to optimize the transmitted information. Second, the signal design could be modified in order to increase the data rate, $R_{d}$, allowing the broadcasting of more data information. Moreover, following this procedure, the transition from the reception of the SBAS L1 signal only to the reception of both SBAS signals, L1 signal and analyzed L5 signal, will be smoother for the receiver manufacturers.

The third and last goal consists in analyzing signal designs which preserve or even improve about the same performance as the current IWG SBAS L5 SIS ICD signal, specially the demodulation performance. Unfortunately, a significant $R_{d}$ increase implies a decrease of the signal performance which can be partially/totally mitigated with advanced signal structure techniques. Therefore, signal structures strongly degrading key IWG SBAS L5 SIS ICD signal performance are discarded.

\section{REQUIREMENTS}

In this section, the requirements imposed for the design of the SBAS L5 signal are described. The requirements are 
derived from the last two motivations of the previous section, $100 \%$ compatibility with the current IWG SBAS L5 SIS ICD definition and the preservation of key IWG SBAS L5 SIS ICD signal performances.

Therefore, we can divide the requirements depending on the motivation which originated them:

- Requirements imposed by the IWG SBAS L5 SIS ICD signal structure and message content which watch over the $100 \%$ compatibility with the IWG SBAS L5 SIS ICD definition.

- $\quad$ Requirements imposed by the IWG SBAS L5 SIS ICD signal performance which watch over the preservation of key IWG SBAS L5 SIS ICD signal performances. These performances can be derived from DO 229 and DO 235B specification.

These requirements are described in detail in sections III.B and III.C but first, a quick description of the IWG SBAS L5 SIS ICD signal structure is given. Finally, note that in order to gain data rate, the signal performance could be degraded. In this case, we will evaluate if the data rate gain compensates the signal performance degradation.

\section{A. IWG SBAS L5 SIS ICD signal structure}

The IWG SBAS L5 SIS ICD signal structure consists in a BPSK modulation with a symbol rate equal to 500 symbols/s. The implemented channel code is the $(171,133)$ convolutional channel code with code rate equal to $1 / 2$ [7]. Therefore, the IWG SBAS L5 SIS ICD signal transmits 250 information bits/s. The information bits are divided in messages of 250 information bits. Each message contains 4 different fields: first 8 bits of preamble which serve to conduct the synchronization message process; second, 6 bits of message ID which serve to define the message purposes; third, 212 data information bits which are the core information of the system; fourth and last, $24 \mathrm{CRC}$ bits which serve to protect the integrity of the entire message (see Fig. 1).

\section{B. IWG SBAS L5 SIS ICD structure and Message content requirements}

The requirements imposed by the IWG SBAS L5 SIS ICD signal structure and message content on the new GEO L5 signal are just for the GEO L5 data component. This means that any data component(s) of the SBAS L5 signal should have the following characteristics:

- An information bit rate of 250 information bits per second $\left(R_{b}=250 \mathrm{~b} / \mathrm{s}\right)$ [7], and a coded bit rate of 500 coded bits per second $\left(R_{c}=500 \mathrm{~b} / \mathrm{s}\right)$ [7]. Consequently, a channel code of rate equal to $1 / 2$ $(r=1 / 2)$ is also imposed [7].

- A BPSK modulation from the demodulation point of view (for example BPSK(10) as in GPS L5). In consequence, the symbol rate is $R_{s}=500 \mathrm{symb} / \mathrm{s}$.
Fig. 1. SBAS information message and SBAS coded message

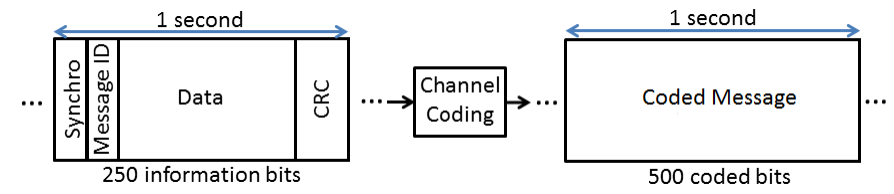

- A BPSK symbol is spanned by a primary PRN code period or by a multiple number of primary PRN code periods.

- An alarm message can interrupt the nominal message at any integer number of seconds (e.g., at 1second, 2 seconds, etc., but not at 2.5 seconds).

- The probability of missed alarm is set at $P_{m d}=10^{-8}$.

\section{IWG SBAS L5 SIS ICD signal performance requirements}

The IWG SBAS L5 SIS ICD signal performance requirements are derived from the SBAS L1 legacy signal. These performances are defined when the SBAS signal is seen as a signal providing geo-ranging and data transmission capabilities. In this paper, these performance requirements are called classic performance requirements.

Additionally, we define a new type of SBAS signal performance requirements where the SBAS signal only provides data transmission and no geo-ranging in order to take into account the future increase of available in-view GNSS constellations which may not make necessary the provision of the geo-ranging service by SBAS. These performance requirements are called alternative performance requirements.

Signal structures candidates will be presented for both signal performance requirements in order to cover both possible future options.

\section{C.1. Classic SBAS signal performance requirements}

The requirements imposed by the classic SBAS L1 legacy signal performance when SBAS signal is used for geo-ranging and data transmission capabilities are:

Acquisition: DO 229 MOPS and DO 235 does not specify an acquisition performance for a SBAS satellite but only require that inside an interval of 2.5 minutes after power recovery the SBAS satellite signal should be acquired, and that verification, data demodulation, achievement of accuracy and integrity requirement must be done [7][8]. Therefore, assuming that the SBAS signal can be acquired within the required time, the performance will be directly given from the acquisition threshold: in IWG SBAS L5 SIS ICD definition for a coherent integration time of $2 \mathrm{~ms}$ and 50 non-coherent integrations (a total of $100 \mathrm{~ms}$ ), the acquisition threshold, for a probability of false alarm $P_{f a}=10^{-4}$, and a probability of detection $P_{d}=$ 0.99 , is equal to $C / N_{0}=28.7 \mathrm{dBHz}$ when an interference of $19 \mathrm{dBHz}$ is present [10].

Tracking: Only the carrier phase tracking is considered in this article. The carrier tracking performance is set from the cycle slip rate. However, DO 229 MOPS does not specify a particular numerical value of cycle slip probability per second [9]. Usually, a value equal to $10^{-4} / \mathrm{s}$ or $10^{-5} / \mathrm{s}$ is chosen for two reasons: in order to guarantee a satisfying code tracking 
accuracy and in order to guarantee the demodulation performance. The $\mathrm{C} / \mathrm{N}_{0}$ required to obtain a cycle slip rate of $10^{-5} / \mathrm{s}$ is about $30 \mathrm{dBHz}$ for IWG SBAS L5 SIS ICD definition [9] (normal dynamics for a constant error of $2.7^{\circ}$ [8], satellite oscillator phase noise of $5.7^{\circ}$ [8], receiver oscillator phase noise defined in [9], one-sided carrier loop bandwidth of $B_{L}=10 \mathrm{~Hz}$, and coherent integration time of $T=2 \mathrm{~ms}$ ).

Demodulation: The SBAS message loss rate, $\mathrm{WER}_{\mathrm{T}}$, should be less than 1 in $1 \mathrm{e} 3$ [7]. This value was derived from the probability of missed alarm requirement, $P_{m d}=10^{-8}$, since the number of consecutive broadcasted alarms and the $\mathrm{WER}_{\mathrm{T}}$ determine $P_{m d}$ (or the other way around):

$$
P_{m d}=10^{-8}=\left(W E R_{T}\right)^{n}
$$

- $\quad n$ is the number of consecutive alarms

Therefore, assuming that at least 3 consecutive alarms are broadcasted (currently this number has been increased to 4 ), the $\mathrm{WER}_{\mathrm{T}}$ should be at least equal to $10^{-3}$.

Moreover, for a pure data component, the $\mathrm{WER}_{\mathrm{T}}$ depends on two factors: on the WER of the implemented channel code, $P_{w}$ or $\mathrm{WER}_{\mathrm{C}}$, and on the cycle slip rate per second, $P_{\text {slip }}$, as previously said. The relationships between these two factors can be expressed as:

$$
W E R_{T}=10^{-3}=1-\left(1-P_{\text {slip }}\right)^{l}\left(1-P_{w}\right)
$$

- $\quad l$ is the number of integer seconds the codeword spans.

For the IWG SBAS L5 SIS ICD definition, the codewords span 1 second. Therefore, assuming a classical cycle slip rate of $10^{-5} / \mathrm{s}$, the $P_{w}$ has to be equal to $10^{-3}$. A $P_{w}=10^{-3}$ is obtained for a $C / N_{0}=29.5 \mathrm{dBHz}$ (same carrier tracking errors as defined before) [9]. However, since this value $C / N_{0}$ is lower than the value necessary to obtain a cycle slip rate of $10^{-}$ $5 / \mathrm{s}$, the demodulation performance threshold is set to $30 \mathrm{dBHz}$.

Summary: The classic performance thresholds for the IWG SBAS L5 SIS ICD definition are defined in TABLE I.

TABLE I. CURRENT SBAS L5 SIGNAL PERFORMANCE THRESHOLDS

\begin{tabular}{|c|c|c|}
\hline Acquisition & Tracking & Demodulation \\
\hline $28.7 \mathrm{dBHz}$ & $30 \mathrm{dBHz}$ & $30 \mathrm{dBHz}$ \\
\hline
\end{tabular}

\section{C.2. Alternative $S B A S$ signal performance requirements}

The requirements defined by the alternative SBAS L1 legacy signal performance (SBAS signal only used for data transmission capabilities) and the reason for the study of this possible future option are given next.

In the previous section, the $P_{\text {slip }}$ traditional value for tracking capabilities was used to determine the value of the channel code WER, $P_{w}$. However, if SBAS signal is mainly used for data transmission capabilities, the code tracking accuracy imposition on the cycle slip rate could be relaxed [9].

In fact, DO 229 MOPS does not specify that the SBAS signal should be used for positioning capabilities, just its design choice makes it possible for SBAS GEOs to operate as additional ranging sources [11]. Therefore, the code tracking accuracy could be slightly degraded without significant effects on the demodulation performance. Moreover, future GPS L5, L1C, GALILEO E1 OS, E5 and other GNSS signals (GLONASS and future BEIDOU) [12][13][14] will incorporate a pilot component which will provide finer synchronization with more accurate pseudo-range measurements. This fact altogether with the expected increase of in-view GNSS satellites (after the deployment of new GNSS constellations) could imply the discarding of the SBAS signal for positioning capabilities; unless a pilot component is implemented for SBAS. Therefore, the SBAS signal data component could only be used for demodulation capabilities.

In this case, equation (3) can be used to find different relationships between $P_{w}$ and $P_{\text {cycle }}$ which could lower the final demodulation performance threshold. For example, for 1 second length codewords, $l=1$, the probabilities values could be $P_{w}=5 \cdot 10^{-4}$ with $P_{\text {slip }}=5 \cdot 10^{-4} \mathrm{slips} / \mathrm{s}$ or $P_{w}=10^{-5}$ with $P_{\text {slip }}=10^{-3} \mathrm{slips} / \mathrm{s}$.

Note that equation (3) can only be used when the occurrence of a cycle slip only affects the decoding of the current received codeword. Therefore, the receiver needs a method which either detects the cycle slips or is not affected by them. In this article, the notion of differential encoding is introduced in order to reduce the impact of a cycle slip just on the codeword being received at the instant of the cycle slip occurrence (see section V.G).

Finally, the alternative the IWG SBAS L5 SIS ICD signal performance thresholds are defined in TABLE II.

TABLE II. ALTERNATIVE SBAS L5 SIGNAL PERFORMANCE THRESHOLDS

\begin{tabular}{|c|c|}
\hline Acquisition & Demodulation \\
\hline $28.7 \mathrm{dBHz}$ & Lower $\mathrm{C} / \mathrm{N}_{0}$ value between $P_{w}$ and $P_{\text {cycle }}$ \\
\hline
\end{tabular}

\section{ADVANCED SIGNAL STRCTURES TECHNIQUES}

In this section, the advanced signal structures techniques proposed to increase the SBAS L5 signal data rate while keeping 100\% compatibility with IWG SBAS L5 SIS ICD definition and their performances are presented.

The advanced signal structure techniques proposed in this article are: CSK modulation, linear block channel codes (LBCC), 1-second length signal blocks, pilot component, synchronization component and $2^{\text {nd }}$ data component. A detailed definition of each one is presented in the following sections.

Finally, one section is provided describing the backward compatibility with the current dispreading PRN codes signal structure since the CSK modulation could modify this structure.

\section{A. Code Shift Keying (CSK) Modulation}

The CSK modulation has as main objective to increase the bit rate of a Direct Sequence Spread Spectrum signal. In this article, the CSK modulation is used to increase the $2^{\text {nd }}$ data component bit rate and to broadcast information on the synchronization component. For a better explanation of a CSK modulation in a GNSS context, the reader is referred to [15]. 
Fig. 2. Example of CSK Modulation with $M=4$ (and $U=2$ bits)

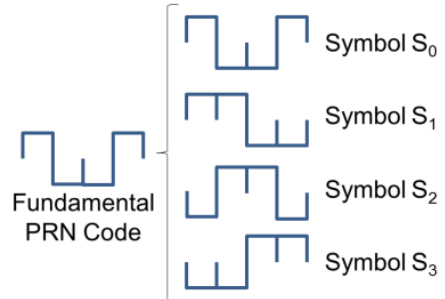

Fig. 3. Codeword source mapping B: all the bits mapped by a CSK symbol belong to the same mesasge

CSK symbol CSK symbol CSK symbol CSK symbol

\begin{tabular}{|l|l|l|l|}
\hline 1 bit & 1 bit & 1 bit & 1 bit \\
\hline 1 bit & 1 bit & 1 bit & 1 bit \\
\hline 1 bit & 1 bit & 1 bit & 1 bit \\
\hline 1 bit & 1 bit & 1 bit & 1 bit \\
\hline
\end{tabular}

1 bit $=$ Message 1

1 bit $=$ Message 2

Fig. 4. Comparison between a BPSK modulated signal and the introduction of a Static CSK $(4,8)$ modulation over the BPSK modulated signal: 4 consecutives BPSK symbols constitute a CSK symbol and 8 bits are mapped with 256 different circular phase shfts of the fundamental PRN code. The bit rate is doubled.

\begin{tabular}{|c|c|c|c|c|c|c|c|}
\hline $\begin{array}{c}\text { BPSK } \\
\text { Symbol }\end{array}$ & BPSK & BPSK & BPSK & BPSK & BPSK & BPSK & BPSK \\
Symbol & Symbol & Symbol & Symbol & Symbol & Symbol & Symbol \\
\hline
\end{tabular}

\begin{tabular}{|l|l|l|l|l|l|l|l|}
\hline $\begin{array}{l}\text { PRN Code } \\
\text { Symbol 0 }\end{array}$ & $\begin{array}{l}\text { PRN Code } \\
\text { Symbol 0 }\end{array}$ & $\begin{array}{l}\text { PRN Code } \\
\text { Symbol 0 }\end{array}$ & $\begin{array}{l}\text { PRN Code } \\
\text { Symbol 0 }\end{array}$ & $\begin{array}{l}\text { PRN Code } \\
\text { Symbol 35 }\end{array}$ & $\begin{array}{l}\text { PRN Code } \\
\text { Symbol 35 }\end{array}$ & $\begin{array}{l}\text { PRN Code } \\
\text { Symbol 35 }\end{array}$ & $\begin{array}{l}\text { PRN Code } \\
\text { Symbol 35 }\end{array}$ \\
\hline
\end{tabular}

CSK modulation is a form of orthogonal $M$-ary modulation over a communication channel [15] since $M$ orthogonal signaling waveforms are used to transmit $U=\log _{2}(M)$ bits. The special characteristic of the CSK modulation with respect to the typical orthogonal $M$-ary signaling is that each waveform (or symbol representing a set of input bits) is obtained from a different circular cyclic phase shift of a fundamental PRN code. Moreover each circular cyclic phase shift is made by an integer number of chips [15] and is assumed to be a full period version of the fundamental PRN code [15]. Fig. 2 provides a graphical explanation of the CSK modulation.

In order to estimate which CSK symbol is transmitted, a matched filter should be implemented for each symbol of the alphabet [15]. For a CSK modulation, since each symbol is a circular shift version of the fundamental PRN code, each matched filter output is equivalent to the evaluation of the correlation between the received signal and the fundamental PRN code at a different shift. The correlation output with the largest positive value determines the transmitted CSK symbol.

In this article, the selected codeword source mapping is mapping B [15], which means that all the bits mapped by a CSK symbol belong to the same message (see Fig. 3).
Finally, two kinds of CSK modulation implementations are presented in this article: Static CSK and Dynamic BPSK/CSK.

\section{A.1. Static CSK implementation}

The traditional implementation of a CSK modulation consists in having a CSK symbol with a fixed length of $N$ consecutives PRN codes which maps a fixed number $U$ of bits. In this article, this implementation is denoted as Static $\operatorname{CSK}(N, U)$.

In the case of a BPSK signal, such as the IWG SBAS L5 SIS ICD signal, the introduction of a static $\operatorname{CSK}(N, U)$ will consists in first selecting the primary PRN code of any BPSK symbol as the fundamental PRN code of the CSK modulation. Second, it will fix a constant CSK symbol length: how many consecutive $N$ BPSK symbols constitute a CSK symbol (the same circular shift will be applied to each one of the primary PRN code of the $N$ consecutive BPSK symbols). Third and last, it will determine the number of bits, $U$, mapped by a CSK symbol $\left(M=2^{U}\right.$ possible circular shifts of the primary PRN code, and $M \leq L$ where $\mathrm{L}$ is the number of chips of the fundamental PRN code). Therefore, the new proposed SBAS L5 $2^{\text {nd }}$ data component signal could contain $500 / N$ symbols/s and could transmit $500 \cdot U / N$ bits/s. For example, for a Static CSK $(4,8)$, the CSK modulated IWG SBAS L5 SIS ICD signal will contain 125 CSK symbols per second (spanning 4 BPSK symbols each) with 256 possible different circular shifts of the primary PRN code. The final bit rate would be $1000 \mathrm{bits} / \mathrm{s}$ and thus the original bit rate would be increased by twice its original value. See Fig. 4 for a graphical explanation.

\section{A.2. Dynamic BPSK/CSK implementation}

The Dynamic $\operatorname{CSK}(N, U)$ implementation consists in dynamically changing the length of the CSK symbol, $N$, (number of consecutive BPSK symbols constituting the CSK symbol) and the number of bits mapped by a CSK symbol, $U$; in other words, changing dynamically among different static CSK configurations. Moreover, if the signal dynamically allows the implementation of a CSK modulation or a BPSK modulation, the implementation is denoted as Dynamic $\operatorname{BPSK} / \operatorname{CSK}(N, U)$.

In this article, 3 different dynamic BPSK/CSK configurations which provide 3 different bit rates are defined for the second data component (see TABLE III. ).

TABLE III. SELECTED DYNAMIC BPSK/CSK CONFIGURATIONS

\begin{tabular}{|c|c|c|c|}
\hline Modulation & BPSK(1) & CSK $(4,10)$ & $\operatorname{CSK}(2,10)$ \\
\hline Increase of bit information rate, $R_{b}$ & $\mathrm{x} 1$ times & $\mathrm{x} 2.5$ times & $\mathrm{x} 5$ times \\
\hline
\end{tabular}

Nevertheless, the final choice is left open for any combination $\operatorname{CSK}(N, U)$ which is desired and which fulfills the signal constraints.

Finally, in order to implement a Dynamic BPSK/CSK modulation, the receiver must now in advance which one out of the 3 configurations is being used at any moment. In this article, this information is transmitted by the synchronization component (see section IV.D). Moreover, each configuration is valid only for 1 -second length block of the signal (see section IV.C). 


\section{B. Linear block channel codes (LBCC)}

The introduction of linear block channel codes (LBCC) instead of the IWG SBAS L5 SIS ICD $(171,155)$ convolutional code $(\mathrm{CC})$ is analyzed in this article.

The main difference between a LBCC and a CC is the number of information bits protected by each code. On one hand, a CC encodes an infinite stream of information bits. Therefore, the message size, or number of information bits protected by the CRC (plus the CRC bits) are not determined by the code but by the application (or system). On the other hand, a LBCC encodes a fixed number of information bits into a group (or block) of output coded bits. This output block is called codeword and determines the message size. In this article, an entire message occupies an entire codeword, as it is usually done, and they are used equivalently from now on.

The analysis of using LBCCs instead of the IWG SBAS L5 SIS ICD CC has been made for three main related reasons. The first reason is that current LBCCs are more powerful than CCs when both families of codes have the same code rate, $r=1 / 2$, and when the message size is of at least 250 information bits [16]. The second reason is that for a LBCC, the increase of the message size implies an improvement of the demodulation threshold whereas for a CC the threshold is degraded [17].

The third reason is that thanks to the possibility of increasing the message size, the number of data information bits to the total number of information bits, data information bits plus CRC bits and header bits, ratio is increased (see equation (1)). For example, if the codeword spans 3 seconds, the number of CRC and header bits is reduced by at least 64 bits: reduction of $2 \mathrm{CRC}$ and 2 synchronization groups of bits since just $1 \mathrm{CRC}$ and 1 group of synchronization bits is required; the message ID can be either maintained or removed. Therefore, the introduction of longer than 1 second codewords allows to improve the demodulation threshold and to increase the data rate, $R_{d}$. See Fig. 5 for a graphical explanation.

There are two main drawbacks of implementing a LBCC. The first one is that the synchronization of a LBCC is more difficult to obtain than for a $\mathrm{CC}$ since the synchronization bits cannot be protected by the code itself: a CC does not need to be synchronized for decoding the received message whereas a LBCC does (CC only needs the synchronization bits for synchro-frame purposes but not for decoding). The second drawback is that the SBAS system requires the possibility of broadcasting alarm messages each $1 \mathrm{~s}$ if necessary. However, since a LBCC has to wait until the reception of the complete message before decoding and thus accessing the information, no messages longer than 250 information bits could be used. This limitation will reduce significantly the advantages of using LBCCs. In order to overcome these two drawbacks, the solution chosen in this article is the implementation of a synchronization or pilot component (see sections IV.D and IV.E) and the division of the signal in 1 second length blocks (see section IV.C).

The LBCCs chosen in this paper are described in more detail in [16]. These codes are CCSDS (Consultative Committee for Space Data System) Telemetry Standard TurboCodes [18] where the interleavers have been replaced with S-random interleavers [19] in order to obtain codewords of a size equal to 500 coded bits or to a multiple of 500 coded bits. The channel codes considered in this article are given in TABLE IV.

\section{Signal division in 1 second length blocks}

In order to allow the compatibility with the IWG SBAS L5 SIS ICD definition and in order to allow the possibility of sending an alarm message every integer number of seconds, the proposed signal structure, and thus the proposed signal components, is always divided in blocks of 1 second length (500 BPSK symbols).

This means that if a message larger than 500 coded bits is broadcasted with a BPSK(1) modulation (see section IV.B), the transmission will be made in blocks of 500 bits. Therefore, the receiver must wait for the reception of all the blocks before decoding the codeword. See Fig. 6 for a graphical example.

The division in blocks of 500 BPSK symbols also means that there are different types of blocks and that the receiver should be able to differentiate them. Moreover, when using a dynamic BPSK/CSK modulation, the selected configuration will only be valid for a block of 500 BPSK symbols. The different types of blocks are: alarm message, CCSDS-250 message, CCSDS-500 first block, CCSDS-500 no-first block (2 blocks make a message), CCSDS-1250 first block and CCSDS1250 no-first block (5 blocks makes a message), $\operatorname{CSK}(4,10)$ block and $\operatorname{CSK}(2,10)$.

The signal structure responsible for allowing the signal block division is either the synchronization component or the pilot component (see sections IV.D and IV.E). The responsible for determining which type of block is broadcasted every 1 second is the synchronization component (see section IV.D).

TABLE IV. LBCCS PROPOSED FOR SBAS L5 SIGNAL

\begin{tabular}{|c|c|c|}
\hline Channel Code & Coded Bits & Information Bits \\
\hline SBAS legacy (171, 133) CC & 500 & 250 \\
\hline CCSDS-250 & 500 & 250 \\
\hline CCSDS-500 & 1000 & 500 \\
\hline CCSDS-1250 & 2500 & 1250 \\
\hline
\end{tabular}

Fig. 5. Header and $\mathrm{CRC}$ bits reduction between different messages lengths.

\begin{tabular}{|c|c|c|c|c|c|c|c|c|}
\hline \multicolumn{3}{|c|}{250 information bits } & \multicolumn{3}{|c|}{250 information bits } & \multicolumn{3}{|c|}{250 information bits } \\
\hline 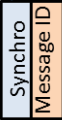 & $\begin{array}{c}\text { Data } \\
\text { (212 data } \\
\text { information bits) }\end{array}$ & 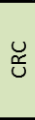 & 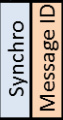 & $\begin{array}{c}\text { Data } \\
\text { (212 data } \\
\text { information bits) }\end{array}$ & & 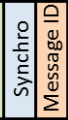 & $\begin{array}{c}\text { Data } \\
\text { ( } 212 \text { data } \\
\text { information bits) }\end{array}$ & $\begin{array}{l}\text { U্ } \\
\text { U. }\end{array}$ \\
\hline \multicolumn{9}{|c|}{750 information bits } \\
\hline 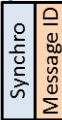 & & & (712c & $\begin{array}{l}\text { Data } \\
\text { lata information bi }\end{array}$ & & & & Ư \\
\hline
\end{tabular}

Fig. 6. SBA L5 signal divided in 1-second length blocks

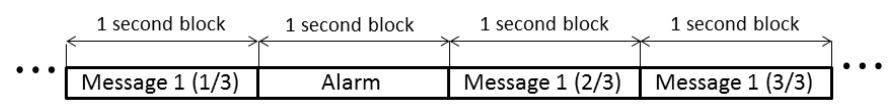




\section{Syncrhonization Component}

The synchronization component is an additional signal component which has to be broadcasted along the data component. The total signal power must thus be shared between at least these two components. The synchronization component has 1 main function and 2 optional functions:

1) (Main function): Determination of the type of transmitted 1-second length signal block (alarm message, LBCC block and dynamic BPSK/CSK).

2) (Optional): Synchronization of the 1-second length blocks of the received signal.

3) (Optional): Determination of a reduced number of types of messages (less than the 6 bits field of the IWG SBAS L5 SIS ICD definition).

The synchronization block can have two different structures depending on whether the first optional function is carried out by the synchronization component or by a pilot component (see section IV.E). These two structures are denoted as simplified and complex and are commented next.

\section{D.1. Simplified synchronization component}

The simplified synchronization component implements a CSK modulation. In this case, the CSK symbol spans the complete length of a 1-second signal block, 500 BPSK symbols. However, as opposite to the case where the data component was CSK modulated, the fundamental PRN code of the CSK modulation is a secondary PRN code implemented over the BPSK symbols instead of the primary PRN code; therefore we have a $\operatorname{CSK}(1, U)$ modulation (see Fig. 7). Finally, depending on the number of bits mapped by the CSK symbol, $U$, a reduced message ID could be transmitted in addition to the type of 1 -second length signal block (since $L=500, U<9)$.

\section{D.2. Complex synchronization component}

The complex synchronization component is divided into two parts. The first part spans the first 250 symbols of a 1second length signal block, is BPSK(1) modulated and implements a secondary code over the BPSK symbols denoted A. The second part spans the last 250 BPSK symbols, implements a secondary PRN code denoted B over the BPSK symbols, and finally applies a CSK modulation over the secondary PRN code B: the secondary PRN code B is the fundamental PRN sequence of the CSK modulation $(\operatorname{CSK}(1, U))$. Moreover, secondary PRN code A is orthogonal to the secondary PRN code B (see Fig. 8).

The first part is thus responsible for synchronizing the 1second length blocks of the signal since once the primary PRN code is acquired, the receiver only needs to find the secondary PRN code of the first synchronization component part to be synchronized with the 1-second length signal blocks. Note that in the ideal case, the receiver should not synchronize with the second synchronization component part since the first and second parts secondary codes are orthogonal. However, a good optimization of the secondary PRN codes must be done in order to avoid a false lock for a bad combination of a partial correlation between the first part secondary PRN code and the second part secondary PRN code (see Fig. 9).
Fig. 7. Simplified synchroniztaion component: CSK modulation over the BPSK symbols secondary code - 2 possible circular cyclic shifts of the CSK modulation apping different sets of $U$ bits.

1 second (500 BPSK symbols)

\begin{tabular}{|c|c|c|c|c|c|c|c|}
\hline$b_{0}$ & $b_{1}$ & $b_{2}$ & $b_{3}$ & $\cdots$ & $b_{497}$ & $b_{498}$ & $b_{499}$ \\
\hline \multicolumn{8}{|c|}{ BPSK Symb } \\
\hline$b_{498}$ & $b_{499}$ & $b_{0}$ & $b_{1}$ & $\cdots$ & $b_{495}$ & $b_{496}$ & $b_{497}$ \\
\hline
\end{tabular}

Fig. 8. Complex synchroniztaion component: $1^{\text {st }}$ Part is fixed and implements a secondary code denoted $\mathrm{A}, 2^{\text {nd }}$ part implements a CSK modulation over the BPSK symbols secondary code - 2 possible circular cyclic shifts of the CSK modulation apping different sets of $U$ bits.

1 second (500 BPSK symbols)

\begin{tabular}{|c|c|c|c|c|c|c|c|c|c|}
\hline \multicolumn{5}{|c|}{ First Part (Fixed - 250 BPSK symbols) } & \multicolumn{5}{|c|}{ Second Part (CSK - 250 BPSK symbols) } \\
\hline$b_{0}$ & $b_{1}$ & $\cdots$ & $b_{248}$ & $b_{249}$ & $b_{0}{ }^{\prime}$ & $b_{1}{ }^{\prime}$ & $\cdots$ & $b_{248}{ }^{\prime}$ & $b_{249^{\prime}}$ \\
\hline \multicolumn{3}{|c|}{ BPSK Symb } & \multicolumn{5}{|c|}{ BPSK Symb } & & \\
\hline$b_{0}$ & $b_{1}$ & $\cdots$ & $b_{248}$ & $b_{249}$ & $b_{248}{ }^{\prime}$ & $b_{249}{ }^{\prime}$ & $\cdots$ & $b_{246}^{\prime}$ & $b_{247^{\prime}}$ \\
\hline
\end{tabular}

Fig. 9. Complex synchroniztaion component correlation. In upper figure the correlation is 0 since secondary PRN codes A and B are orthogonal. In lower figure, the correlation is the sum of two partial correlations: PRN code A with shifted PRN code A, and PRN code B with shifted PRN code A.
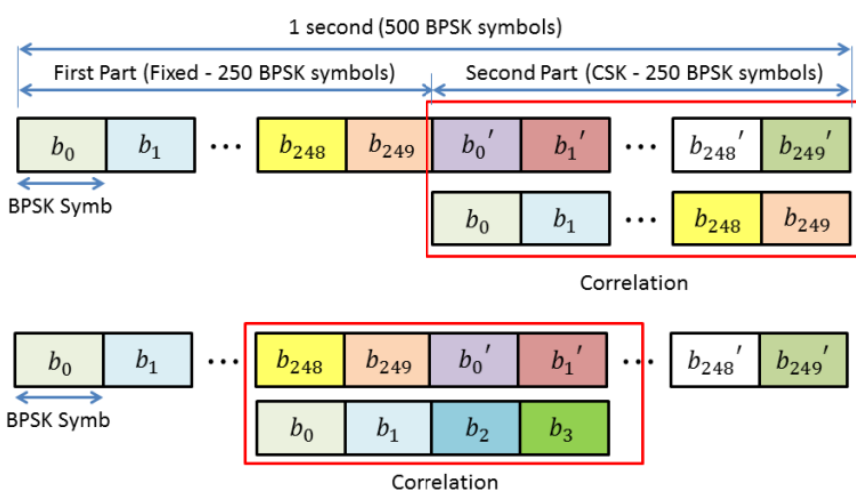

Additionally, in order to further mitigate this effect, a rotation of the primary PRN code of the second part of the synchronization component could be done; however this solution would make the receiver more complex and would change the spreading structure of the signal.

Finally, the second synchronization component part is responsible for determining the type of 1-second length signal block being transmitted and for transmitting a reduced message ID (since $L=250, U<8$ ).

\section{E. Pilot Component}

The pilot component implements first a primary PRN code with the same length as the data component primary PRN code and second, it implements a secondary PRN code of $1 \mathrm{~s}$ of period (and thus 500 chips) over the primary PRN code.

The introduction of a pilot component is designed in order to conduct 2 main functions and 1 optional function: 
Fig. 10. Example of coordination between 2 data components implementing LBCCs.Coherent accumulation of the integrity message broadcasted simultaneoulsy by the two data components.

Synchronization Component
\begin{tabular}{|c|c|c|}
\hline LBCC CCSDS-500 ID & Integrity ID & $\operatorname{CSK}(4,10)$ ID \\
\hline 1st Data Component & \\
\hline CCSDS-500 message (1/2) & Integrity Message & CCSDS-1500 message $(1 / 5)$ \\
\hline 2nd Data Component & & \\
\hline CCSDS-500 message (2/2) & Integrity Message & $\operatorname{CSK}(4,10)$ \\
\hline & \multicolumn{2}{|c|}{ Coherent Accumulation } \\
\cline { 2 - 3 }
\end{tabular}

Fig. 11. Two data components implementation adapted for legacy users: legacy users only employ the $1^{\text {st }}$ data component having the IWG SBAS L5 SIS ICD signal structure; advanced users take advantages of the two data components.

Synchronization Component

\begin{tabular}{|l|l|l|}
\hline CCSDS-500 ID (1st part) & Integrity ID & CCSDS-500 ID (non 1st part) \\
\hline
\end{tabular}

\begin{tabular}{|c||c|c|}
\hline 1st Data Component & \multicolumn{1}{c|}{} \\
\hline SBAS legacy signal & SBAS legacy signal Integrity & SBAS legacy signal \\
\hline 2nd Data Component & & \\
\hline CCSDS-500 message (1/2) & SBAS legacy signal Integrity & CCSDS-500 message (2/2) \\
\hline & Coherent Accumulation \\
\hline
\end{tabular}

Advanced Users

1) (Main): Synchronization of the 1-second length blocks of the received signal.

2) (Main): Mitigation or removal of the cycle slips influence.

3) (Optional): Providing of geo-ranging capabilities

Note that the 1-second length signal blocks synchronization by the pilot component allows the simplified synchronization component implementation (and thus more bits can be transmitted by the synchronization component).

The introduction of a pilot component mitigates the influence of the cycle slips impact since the probabilities of occurrence are obtained at a much lower $\mathrm{C} / \mathrm{N}_{0}$ [20][21]. Besides, if the SBAS signal only has to provide data transmission capability (see section III.C.2), the introduction of a pilot component completely removes the cycle slip rate impact since a phase jump of $2 \pi$ does not influence the BPSK demodulation process.

Finally, the pilot component must share the total signal power with the data component and the synchronization component. However, if the pilot component is also designed for geo-ranging capabilities (see section III.C.1), we assume that additional power equal to the original signal power amount will be completely allocated to the pilot component.

\section{F. $2^{\text {nd }}$ Data Component}

The introduction of a $2^{\text {nd }}$ data component is designed for 2 main functions:

1) Increase of the bit rate, $R_{b}$.

2) Compatibility with the IWG SBAS L5 SIS ICD definition.

The introduction of a $2^{\text {nd }}$ data component allows in theory to double the bit rate, $R_{b}$. However, this also implies that the demodulation performance thresholds are about $3 \mathrm{~dB}$ lower than when only one data component is implemented since the power is shared. For some information, this degradation of performance is not desired, for example for the alarm/integrity information. Therefore, this article proposes that when some sensitive information is transmitted, the two data components carry the same information. Then, the receiver only needs to coherently combine the two data components in order to recover the original demodulation performance. The information necessary to coordinate the two data components will be given by the synchronization component and by preestablished rules: when an integrity message is sent, the receiver knows by reading the information of the synchronization component that both data components carry the integrity message and thus knows that they must be coherently added (see Fig. 10).

Finally, the second data component is also designed in order to allow the receiver manufacturers the choice of using just one or the two data components. The first data component could implement the IWG SBAS L5 SIS ICD signal structure and the second data component could implement the proposed LBCCs and dynamic CSK modulation. Therefore, some users could decide to use only the first component with a classical receiver without any concerns by the other signal components but with degraded performances, and other users could opt for using the full capacity of the SBAS L5 signal with a more complex receiver which will use all the signal components (see Fig. 11). Nevertheless, we will show that the use of LBCCs in the first data component is necessary to overcome the performance degradation of using only the first data component (see sections VI.D and VI.E).

\section{G. PRN code structure backward compatibility}

One of the main concerns of the proposed advanced signal structure techniques is whether they present a backward compatibility with the current PRN code structure or not, and thus, if they are compatible with the current 500 bps signal structure.

In fact, the only advanced signal structure which could modify the legacy PRN code structure is the introduction of a CSK modulation. However, the use of a CSK modulation on the synchronization component does not modify the IWG SBAS L5 SIS ICD PRN code structure since the CSK modulation is implemented over the secondary code and not over the primary PRN code. Therefore, among the proposed advanced signal structures, only the introduction of a CSK modulation on a data component in order to increase the final bit rate would modify the IWG SBAS L5 SIS ICD PRN code structure. In any case, the introduction of a CSK modulation would only be considered on the second data component (see section VI.F) and thus, the receiver can always choose to ignore the 1-second length signal blocks containing the CSK modulation (identified using the synchronization component) or simply to completely ignore the $2^{\text {nd }}$ data component.

\section{SIGNAL PERFORMANCES}

In this section, the performance calculations of the different advanced signal techniques presented in the previous section are calculated. The calculation of these performances will be 
used in order to compare the new proposed signal structures with the IWG SBAS L5 SIS ICD signal structure performance.

\section{A. Primary PRN code acquisition}

The performance of the primary PRN code acquisition is not calculated in this article but just some comments are made using as basis [10]. The primary PRN code acquisition threshold is degraded when the signal power is split in several components and all of them are used (integration time limited to $2 \mathrm{~ms}$ ). However, if a pilot component is used, the degradation is fairly limited when the integration time is extended, for example to $\mathrm{T}=20 \mathrm{~ms}$. Therefore, the final primary PRN code acquisition is expected to be degraded but a larger noncoherent integration time will mitigate this effect [10]. Obviously, if a pilot component with additional power is implemented, the primary PRN code acquisition threshold will be significantly improved.

\section{B. Carrier Phase estimation Error}

The carrier phase estimation is conducted over different signal components depending on the chosen signal structure candidate. The choice will follow the logic presented below:

Pilot component is available?

- Yes: Use pilot component

- No: 2 data components are available?

- Yes: Use the 2 data components

- No: Use the only data component

The carrier phase estimation error, $\varepsilon_{\varphi}$, can be modelled as the addition of two terms, a constant term, $\gamma$, representing the error introduced by the receiver dynamics and random variable term, $\varphi$, representing the carrier tracking jitter of the implemented tracking loop. $\varepsilon_{\varphi}$ can thus be modeled as:

$$
\varepsilon_{\varphi}=\gamma+\varphi
$$

$\varphi$ is centered and follows a distribution given in [9] which is completely defined by its variance:

$$
p_{\varphi}(\varphi)=\frac{1}{2 \pi I_{0}\left(1 / \sigma_{\varphi}^{2}\right)} \exp \left(\frac{\cos \varphi}{\sigma_{\varphi}^{2}}\right)
$$

- $I_{n}(x)$ is the $\mathrm{n}^{\text {th }}$ order modified Bessel function of the first kind of $x$.

The carrier tracking jitter variance of the tracking loop, $\sigma_{\varphi}^{2}$, can be expressed as [9]:

$$
\sigma_{\varphi}^{2}=\sigma_{\varphi, \text { noise }}^{2}+\sigma_{\varphi, \text { osc }}^{2}
$$

- $\sigma_{\varphi, \text { noise }}^{2}$ : Contribution to the carrier tracking jitter variance due to thermal noise/interference.

- $\sigma_{\varphi, o s c}^{2}$ : Contribution to the carrier tracking jitter variance due to the oscillator phase noise (both from receiver and satellite).

The expression of $\sigma_{\varphi, \text { noise depends on the signal }}^{2}$ components where the carrier is tracked. For 1 data component (using a Costas Loop) [9]:

$$
\sigma_{\varphi, \text { noise }}^{2}=\frac{\mathrm{B}_{\mathrm{L}}}{\mathrm{C} / \mathrm{N}_{0}}\left(1+\frac{1}{2 \mathrm{C} / \mathrm{N}_{0} \mathrm{~T}}\right)
$$

For two data components having the same power (using a Costas Loop):

$$
\sigma_{\varphi, \text { noise }}^{2}=\frac{\mathrm{B}_{\mathrm{L}}}{\mathrm{C} / \mathrm{N}_{0}}\left(1+\frac{1}{\mathrm{C} / \mathrm{N}_{0} \mathrm{~T}}\right)
$$

For the pilot component (using a $\mathrm{Q}$ discriminator) [20]:

$$
\sigma_{\varphi, \text { noise }}^{2}=\frac{\mathrm{B}_{\mathrm{L}}}{\mathrm{C} / \mathrm{N}_{0}}
$$

- $\mathrm{B}_{\mathrm{L}}$ is the one-sided carrier loop bandwidth

- $\mathrm{T}$ is the coherent integration

- $\mathrm{C}$ is the power of the signal component

$$
\frac{\mathrm{C}}{\mathrm{N}_{0}}=\frac{\mathrm{C}}{\mathrm{N}_{0_{T}}}+10 \cdot \log 10(R)
$$

- $\mathrm{C} / \mathrm{N}_{0, \mathrm{~T}}$ is the total signal $\mathrm{C} / \mathrm{N}_{0}$

- $R$ is the percentage of power allocated to the component being tracked.

The expression of $\sigma_{\varphi, \text { osc }}^{2}$ can be calculated as [9]:

$$
\sigma_{\varphi, o s c}^{2}=\sigma_{\varphi, s a t, o s c}^{2}+\sigma_{\varphi, r x, o s c}^{2}+\sigma_{\varphi, r x, v i b}^{2}
$$

- $\sigma_{\varphi, s a t, o s c}^{2}$ : Contribution to the carrier tracking jitter variance due to the satellite oscillator phase noise.

- $\sigma_{\varphi, r x, o s c}^{2}$ : Contribution to the carrier tracking jitter variance due to the receiver Allen deviation oscillator phase noise.

- $\sigma_{\varphi, r x, v i b}^{2}$ : Contribution to the carrier tracking jitter variance due to the receiver vibration-induced oscillator phase noise.

The values of $\sigma_{\varphi, r x, o s c}^{2}$ and $\sigma_{\varphi, r x, v i b}^{2}$ do not depend on the tracked signal component, and their expressions and their numerical values used in this article are defined in [9]. The numerical value of $\sigma_{\varphi, \text { sat,osc }}$ is defined by DO $235 \mathrm{~B}$ [8] and is equal to $0.1 \mathrm{rad}\left(5.7^{\circ}\right)$ at $B_{L}=10 \mathrm{~Hz}$.

Finally, the constant phase tracking error, $\gamma$, due to the dynamics of the aircraft is equal to [21]:

$$
\gamma=2 \pi \frac{T^{3}}{K_{3}} \cdot \frac{g}{\lambda} \cdot j_{\max }(\mathrm{rad})
$$

- $j_{\max }$ is the maximum jerk (in $\mathrm{g} / \mathrm{s}$ ) experienced by the user receiver. In normal dynamics for an aircraft, $j_{\max }=0.25 \mathrm{~g} / \mathrm{s}$.

- $T$ is the coherent integration

- $\mathrm{g}$ is the gravity acceleration

- $\lambda$ is the wavelength of the carrier signal frequency

- $K_{3}$ is the coefficient given by [21] in their description of the discrete-update PLL 
Note that the constant error phase due to dynamics defined in equation (12) provides a different value than the value presented in section III.C. 1 for the same jerk, $j_{\max }$. From now on, we will use equation (12) to calculate the constant error phase due to dynamics since the expression used in [8][9] are approximations valid for $\mathrm{B}_{\mathrm{L}} T \ll 1$. In fact, equation (12) depends on $\mathrm{T}$ whereas the expression used in [8][9] does not.

\section{Carrier Phase loss of lock}

The PLL loses its lock when the carrier phase estimation error, $\varepsilon_{\varphi}$, is longer than the linear region of the used discriminator. The expression providing the loss of lock is [20]:

$$
\frac{L_{\varphi}}{2} \geq 3 \sigma_{\varphi}+\gamma
$$

- $L_{\varphi}$ is the two-sided discriminator linear region. $L_{\varphi}=180^{\circ}$ for $\mathrm{Q}$ discriminator and $L_{\varphi}=90$ for Costas discriminator.

D. Demodulation power loss due to the carrier phase estimation error

The carrier phase estimation error, $\varepsilon_{\varphi}$, implies a power loss of the used to demodulate the data components. The available $\mathrm{C} / \mathrm{N}_{0}$ for the demodulation, $\mathrm{C} / \mathrm{N}_{0, \mathrm{Du}}$ can be modeled as [8]:

$$
{\frac{C}{N_{0}}}_{D u} \geq \frac{C}{N_{0}} \cdot \cos ^{2}\left(\varepsilon_{\varphi}\right)
$$

- $\mathrm{C} / \mathrm{N}_{0, \mathrm{D}}$ is the $\mathrm{C} / \mathrm{N}_{0}$ available at the data component

In this article, two cases of demodulation power loss which cover the worst case scenario and a normal case scenario are inspected (after evaluating the probability density function of equation (5)):

- Normal scenario: $\varepsilon_{\varphi}=\gamma+\sigma_{\varphi}$

- Worst scenario: $\varepsilon_{\varphi}=\gamma+3 \sigma_{\varphi}$

Therefore, the demodulation performance threshold will be presented taking into account a carrier phase estimation error equal to the value of the normal scenario and worst scenario.

\section{E. Cycle slip rate}

The probability of having a cycle slip in $t$ seconds can be expressed as [9]:

$$
P_{\text {slip }}(t)=1-e^{-t / \bar{T}}
$$

Where $\bar{T}$ is the mean time between cycle slips. The value of $\bar{T}$ depends on the implemented discriminator. For a Costas discriminator [9]:

$$
\bar{T}=\frac{\pi}{4 B_{L} \gamma} \tanh \left(\frac{\pi \gamma}{2 \sigma_{\varphi}^{2}}\right)\left[I_{0}^{2}\left(\frac{1}{4 \sigma_{\varphi}^{2}}\right)+2 \sum_{n=1}^{\infty}(-1)^{n} \frac{I_{n}^{2}\left(\frac{1}{4 \sigma_{\varphi}^{2}}\right)}{1+\left(2 n \sigma_{\varphi}^{2} / \gamma\right)^{2}}\right]
$$

For a $\mathrm{Q}$ discriminator [21]:

$$
\bar{T}=\frac{\pi}{2 B_{L} \gamma} \tanh \left(\frac{\pi \gamma}{\sigma_{\varphi}^{2}}\right)\left[I_{0}^{2}\left(\frac{1}{\sigma_{\varphi}^{2}}\right)+2 \sum_{n=1}^{\infty}(-1)^{n} \frac{I_{n}^{2}\left(\frac{1}{\sigma_{\varphi}^{2}}\right)}{1+\left(n \sigma_{\varphi}^{2} / \gamma\right)^{2}}\right]
$$

\section{F. BPSK demodulation threshold}

The BPSK demodulation threshold in this paper is calculated from the $E_{b} / N_{0}$ necessary to obtain a given $W E R_{c}$ and from the demodulation power loss. The expression is given below:

$$
\frac{\mathrm{C}}{\mathrm{N}_{0}}=\frac{E_{b}}{N_{0}}-10 \cdot \log 10\left(\frac{R \cdot \cos ^{2}\left(\varepsilon_{\varphi}\right)}{250}\right)
$$

- $R$ is the power allocated to the data component.

The $E_{b} / N_{0}$ for obtaining a $W_{c}=10^{-3}$ for the different inspected channel codes is given in TABLE $\mathrm{V}$. The values of the $\mathrm{WER}_{\mathrm{c}}=10^{-3}$ column has been extracted from [16]. The values of the $\mathrm{WER}_{\mathrm{c}}=10^{-5}$ column has been extrapolated from the $\mathrm{WER}_{\mathrm{c}}$ slope of similar codes and from the $\mathrm{WER}_{\mathrm{c}}=10^{-3}$ column values.

TABLE V. CHANNEL CODES DEMODULATION PERFROMANCE PROPOSED FOR SBAS L5 SIGNAL

\begin{tabular}{|c|c|c|}
\hline Type of Channel Coding & $\begin{array}{c}\mathbf{E}_{\mathbf{b}} / \mathbf{N}_{\mathbf{0}} \text { necessary for } \\
\mathbf{W E R}_{\mathbf{c}}=\mathbf{1 0}^{-3}\end{array}$ & $\begin{array}{c}\mathbf{E}_{\mathbf{b}} / \mathbf{N}_{\mathbf{0}} \text { necessary for } \\
\mathbf{W E R}_{\mathbf{c}}=\mathbf{1 0}^{-3}\end{array}$ \\
\hline SBAS L5 (171, 133) CC & 4.2 & 5.6 \\
\hline CCSDS-250 & 2.5 & 3.5 \\
\hline CCSDS-500 & 1.9 & 2.4 \\
\hline CCSDS-1250 & 1.45 & 1.9 \\
\hline
\end{tabular}

\section{G. BPSK demodulation threshold impacted by cycle slips}

In section III.C.2, we discussed about the impact of a cycle slip over the demodulation of a message. In that section, it was stated that the cycle slip only affects the message which is currently being received (some of the bits will be inversed) but not the following messages. However, we said that a method correcting the cycle slips should be implemented.

In this article, we propose a kind of differential encoding/decoding method. For a LBCC, the receiver just executes:

1) LBCC decoding + CRC-24Q verification.

a. Verification succeeds: End

b. Verification fails: Inverse the soft input polarity of the decoding block. Go to 2).

2) LBCC decoding + CRC-24Q verification.

a. Verification succeeds: Cycle slip detect + End

b. Verification fails: Transmission Error

The probability that the message issued by the LBCC decoding pass the verification is very low: at most equal to $1.1921 \mathrm{e}-007$. Therefore, the probability of having a cycle slip false alarm due to step $2 \mathrm{a}$ is very low.

Finally, for the SBAS L5 CC, we take advantage of two facts. First, if from a sequence of information bits $A$, a sequence of coded bits $B$ is generated, from the decoding of the polarity inverted sequence of code bits $B$, denoted as $\bar{B}$, the sequence of information bits being obtained, $C$, is exactly the same sequence of information bits $A$ but with the polarity 
inverted, $\bar{C}=A$ (verified by channel code construction and from simulations). Second, an error pattern modeling a change of polarity is a vector of ones, and this error vector is always detected by the CRC-24Q verification (verified from simulations). Therefore, the method for the legacy $\mathrm{CC}$ is:

1) CC decoding + CRC-24Q verification.

a. Verification succeeds: End

b. Verification fails: Inverse the output bits polarity. Go to 2).

2) CRC-24Q verification.

a. Verification succeeds: Cycle slip detect + End

b. Verification fails: Transmission Error

\section{H. CSK demodulation threshold for the data component}

The CSK demodulation thresholds for the data component are calculated equivalently as for the BPSK demodulation thresholds. The only difference is that equation (18) should take into account the number of BPSK symbols constituting a CSK symbol and the number of bits mapped by a CSK symbol:

$$
\frac{\mathrm{C}}{\mathrm{N}_{0}}=\frac{E_{b}}{N_{0}}-10 \log 10\left(\frac{R \cdot \cos ^{2}\left(\varepsilon_{\varphi}\right)}{250}\right)-10 \log 10\left(\frac{N}{U}\right)
$$

For practical purposes, the LBCC chosen to be implemented in a CSK modulation is the subframe 2 GPS L1C LDPC channel code. The reason is the lack of $\mathrm{E}_{\mathrm{b}} / \mathrm{N}_{0}$ demodulation values for an implementation of the proposed CCSDS LBCC TurboCodes in a CSK modulation. Therefore, since the objective of this paper is to analyze new signal structure candidates open to discussion and optimizations, it was preferred to show real tested demodulation values.

Two $\mathrm{E}_{\mathrm{b}} / \mathrm{N}_{0}$ values are shown in TABLE VI. for a $\mathrm{WER}_{\mathrm{c}}=$ $10^{-3}$ using classical CSK decoding and BICM-ID methods [15]. BICM-ID outperforms classical decoding but requires a more complex receiver.

TABLE VI. SUBFRAME 2 GPS L1C LDPC WITH CSK DEMODULATION PERFORMANCE $\left(E_{b} / N_{0}\right.$ NECESSARY TO OBTAIN WER $\left.=10^{-3}\right)$

\begin{tabular}{|c|c|}
\hline Classical CSK Decoding & BICM-ID \\
\hline 2.6 & 2.15 \\
\hline
\end{tabular}

Finally, note that subframe 2 GPS L1C LDPC has a length of 1200 coded bits. Therefore, when using the first and second dynamic CSK configurations, there are respectively 50 and 100 coded bits which will not be used. Obviously, this loss of performance should be corrected on the final signal design by choosing different LBCCs.

\section{CSK demodulation threshold for the synchronization component}

The CSK modulation implemented on the synchronization component does not contain any channel code. Therefore, its demodulation performance can be calculated directly from the hard demodulation expression of an orthogonal M-ary modulation. Moreover, the final demodulation performance must take into account the allocation of power and the number of BPSK symbols spanned by the CSK modulated part (see section IV.D.2). The demodulation performance expression is:

$$
\frac{\mathrm{C}}{\mathrm{N}_{0}}=\frac{E_{S}}{N_{0}}-10 \log 10\left(T_{C S K} \cdot R \cdot \cos ^{2}\left(\varepsilon_{\varphi}\right)\right)
$$

- $E_{S} / N_{0}$ is the symbol energy to power density noise ratio necessary to obtain a given $P_{s}$ (symbol error probability) of an orthogonal M-ary modulation.

- $\quad T_{C S K}$ is the percentage of time, either 1 or 0.5 , that the CSK modulated part occupies on the synchronization component.

The expression of $E_{S} / N_{0}$ as a function of the desired $P_{S}$ for an orthogonal M-ary modulation is equal to [17]:

$$
\begin{gathered}
P_{S}=1-P_{c} \\
P_{c}=\frac{1}{\sqrt{2 \pi}} \int_{-\infty}^{+\infty}\left(\frac{1}{\sqrt{2 \pi}} \int_{-\infty}^{y} e^{-\frac{x^{2}}{2}} d x\right)^{M-1} e^{-\frac{1}{2}\left(y-\sqrt{\frac{2 E_{S}}{N_{0}}}\right)^{2}} d y
\end{gathered}
$$

- $\quad \mathrm{M}$ is the number of possible symbols $\left(M=2^{U}\right)$, and $U$ is the number of mapped bits.

In this article, since the information carried by the synchronization component is very significant, we suggest a $P_{S}=10^{-8}$ (same level as the probability of missed alarm).

Finally, if we want to make the synchronization component CSK modulated part insensitive to the cycle slips (when the cycle slip has happened before the reception of the synchronization component part), we need just to adjust the CSK demodulation to choose the symbol with the largest absolute value. In this case, the $P_{s}=1-P_{c}$ expression is:

$$
P_{c}=\frac{1}{\sqrt{2 \pi}} \int_{-\sqrt{\frac{2 E_{S}}{N_{0}}}}^{+\infty}\left(\frac{1}{\sqrt{2 \pi}} \int_{-\left(y+\sqrt{\frac{2 E_{S}}{N_{0}}}\right)}^{y+\sqrt{\frac{2 E_{S}}{N_{0}}}} e^{-\frac{x^{2}}{2}} d x\right)^{M-1} e^{-\frac{y^{2}}{2}} d y
$$

\section{J. Secondary PRN code acquisition}

The acquisition performance of the secondary PRN code of either the pilot component or the synchronization component is inspected in this section. The acquisition performance presented in this article is the probability of not being able to synchronize the secondary PRN code after a complete sweep of the entire search range: one cell to inspect for each secondary PRN code chip. This probability is denoted as $P_{2 a c q}$.

The main assumption made in this article is that once the primary PRN code has been acquired, the carrier tracking phase is conducted. Therefore, the acquisition of the secondary PRN code becomes equivalent to the demodulation of a biorthogonal M-ary modulation (equation (23), since cycle slip event correction is not yet performed). For both, the complex synchronization component and the pure pilot component, the parameter $\mathrm{M}$ of equation (23) is equal to 500; the only difference between them is the parameter $T$ described in equation (24).

Moreover, $K$ consecutive secondary PRN codes could be coherently accumulated (non-coherent accumulation is not inspected in this article). 
Therefore, the acquisition performance threshold of the secondary PRN code is given by:

$$
\frac{\mathrm{C}}{\mathrm{N}_{0}}=\frac{E_{S}}{N_{0}}-10 \log 10\left(\frac{T \cdot R \cdot \cos ^{2}\left(\varepsilon_{\varphi}\right)}{K}\right)
$$

- $E_{s} / N_{0}$ is the symbol energy to power density noise ratio necessary to obtain a given $P_{2 a c q}$ (equation (23)).

- $K$ is the number of consecutive accumulated secondary PRN codes.

- $T$ is the percentage of time that the secondary PRN code occupies: 1 for a pilot component and 0.5 for the complex synchronization component.

In this article, the probability of not being able to synchronize the secondary PRN code is set to $P_{2 a c q}=10^{-5}$.

\section{Signal StRUCtURES CANDIDATES}

In this section, the signal structures candidates identified in this paper are presented and their performances are given.

The identified signal structure candidates can be classified into three types depending on the number of data components being implemented and on the use of the IWG SBAS L5 SIS ICD signal structure. Additionally, the performances of the IWG SBAS L5 SIS ICD signal structure are presented as a benchmark for comparison with the other signal structure candidates. Therefore, there are 4 main types of signal structure candidates: 1 data component implementing the IWG SBAS L5 SIS ICD structure, 1 data component implementing new LBCCs, 2 data components with one of them implementing the IWG SBAS L5 SIS ICD structure and the other implementing new LBCCs, and 2 data components implementing new LBCCs. Moreover, for each type of signal structure candidate, 3 options are considered depending on the interpretation of the cycle slip rate limitation (see section III.C.2): classic or alternative cycle slip performance and additional power for geo-ranging capabilities (the final signal has twice the total amount of power). TABLE VII. summarizes the analyzed candidates and the notations given to each one of them. TABLE VIII. summarizes the signal structure candidates and the allocation of power given to each signal component.

In this section, first the signal performances being compared are described and second, the candidates' individual performances are provided. Moreover, the special characteristics of each type of candidate are explained. Finally, the increase of data rate obtained with the use of a dynamic CSK modulation on the $2^{\text {nd }}$ data components is presented

\section{A. Analyzed signal performances}

Acquisition: Primary PRN code acquisition performance is not provided but the reader should expect an acceptable degradation as explained in section V.A. Non-coherent integrations should mitigate this degradation.

Tracking: The $\mathrm{C} / \mathrm{N}_{0}$ threshold required to obtain a $P_{\text {slip }}=$ $10^{-5} / s$ is provided except for the alternative-cycle-slipperformance candidates. Remember that for these candidates the geo-ranging capabilities are not provided.
Demodulation: The demodulation threshold of the identified candidates depends on their structure. This means that the demodulation threshold will be the highest $\mathrm{C} / \mathrm{N}_{0}$ among the following actions (if required by the candidate):

- Secondary PRN code acquisition of the pilot or synchronization component, with $P_{2 a c q}=10^{-5}$.

- Synchronization component demodulation, $P_{S}=10^{-5}$

- PLL loss of lock

- Channel code WER: $P_{w}=W E R_{c}=10^{-3}$ for classical $P_{\text {slip }}$ performance and additional power for georanging capabilities cases, and a variable $W E R_{c}$ for alternative $P_{\text {slip }}$ performance (equation (3)).

- Cycle slip rate: $P_{\text {slip }}=10^{-5} / \mathrm{s}$ for classical $P_{\text {slip }}$ performance and additional power for geo-ranging capabilities cases, and a variable $P_{\text {slip }}$ for alternative $P_{\text {slip }}$ performance (equation (3)).

Signal data rate, $R_{d}$ : Expected data rate increase with respect to IWG SBAS L5 SIS ICD signal is presented.

Finally, the highest threshold between the tracking and demodulation performance will determine the system threshold and conclusions will be extracted from this threshold.

TABLE VII. SIGNAL StRUCTURES CANDIDATES NOTATION SUMMARY

\begin{tabular}{|c|c|c|c|}
\cline { 2 - 4 } \multicolumn{1}{c|}{} & $\begin{array}{c}\text { Classic cycle } \\
\text { slip rate } \\
\text { performance }\end{array}$ & $\begin{array}{c}\text { Alternative } \\
\text { cycle slip } \\
\text { performance }\end{array}$ & $\begin{array}{c}\text { Additional power } \\
\text { for geo-ranging } \\
\text { capabilities }\end{array}$ \\
\hline $\begin{array}{c}\mathbf{1} \text { data } \\
\text { component: } \\
\text { legacy signal }\end{array}$ & $\begin{array}{c}\mathrm{D} 1.1 \\
\text { Power }=100 \%\end{array}$ & $\begin{array}{c}\mathrm{D} 1.2 \\
\text { Power }=100 \%\end{array}$ & $\begin{array}{c}\mathrm{D} 1.3 \\
\text { Power }=200 \%\end{array}$ \\
\hline $\begin{array}{c}\mathbf{1} \text { data } \\
\text { component: } \\
\text { LBCCs }\end{array}$ & $\begin{array}{c}\mathrm{D} 2.1 \\
\text { Power }=100 \%\end{array}$ & $\begin{array}{c}\mathrm{D} 2.2 \\
\text { Power }=100 \%\end{array}$ & $\begin{array}{c}\mathrm{D} 2.3 \\
\text { Power }=200 \%\end{array}$ \\
\hline $\begin{array}{c}\mathbf{2} \text { data } \\
\text { components: } \\
\text { legacy signal } \\
\text { and LBCCs }\end{array}$ & Power $=100 \%$ & Power $=100 \%$ & $\begin{array}{c}\mathrm{D} 3.3 \\
\text { Power }=200 \%\end{array}$ \\
\hline $\begin{array}{c}\mathbf{2} \text { data } \\
\text { components: } \\
\text { LBCCs }\end{array}$ & $\begin{array}{c}\mathrm{D} 4.1 \\
\text { Power }=100 \%\end{array}$ & $\begin{array}{c}\mathrm{D} 4.2 \\
\text { Power }=100 \%\end{array}$ & $\begin{array}{c}\mathrm{D} 4.3 \\
\text { Power }=200 \%\end{array}$ \\
\hline
\end{tabular}

TABLE VIII. SIGNAL StRUCTURES CANDIDATES STRUCTURE AND POWER SUMMARY

\begin{tabular}{|c|c|c|c|c|c|}
\hline Candidate & $\mathbf{1}^{\text {st }}$ Data & $\mathbf{2}^{\text {nd }}$ Data & Pilot & $\begin{array}{c}\text { Simplified } \\
\text { Synchro }\end{array}$ & $\begin{array}{c}\text { Complex } \\
\text { Synchro }\end{array}$ \\
\hline D1.1 & $100 \%$ & -- & -- & -- & -- \\
\hline $\boldsymbol{D} 1.2$ & $100 \%$ & -- & -- & -- & -- \\
\hline $\boldsymbol{D} 1.3$ & $100 \%$ & -- & $\mathbf{1 0 0} \%$ & -- & -- \\
\hline $\boldsymbol{D} 2.1$ & $60 \%$ & -- & $30 \%$ & $10 \%$ & -- \\
\hline $\boldsymbol{D} 2.2$ & $85 \%$ & -- & -- & -- & $15 \%$ \\
\hline $\boldsymbol{D} 2.3$ & $85 \%$ & -- & $\mathbf{1 0 0} \%$ & $15 \%$ & -- \\
\hline $\boldsymbol{D} 4.1$ & $35 \%$ & $35 \%$ & $20 \%$ & $10 \%$ & -- \\
\hline D3.1/D4.2 & $42.5 \%$ & $42.5 \%$ & -- & -- & $15 \%$ \\
\hline D3.3/D4.3 & $45 \%$ & $45 \%$ & $100 \%$ & $10 \%$ & -- \\
\hline
\end{tabular}


TABLE IX. D1 CANDIDATES PERFORMANCE SUMMARY. WER $\mathrm{W}_{\mathrm{C}}=10^{-3} \mathrm{FOR}$ BLUE CELLS AND WER $\mathrm{W}_{\mathrm{C}}=10^{-5}$ FOR GREEN CELLS. $P_{\text {slip }}=10^{-5} / \mathrm{s}$ FOR ORANGE CELLS AND $P_{\text {slip }}=10^{-3} / \mathrm{s}$ FOR VILOET CELLS.

\begin{tabular}{|c|c|c|c|c|}
\hline Candidate & \multicolumn{2}{|c|}{$\begin{array}{c}\text { Data component, } \boldsymbol{W E R}_{c} \\
\left(\mathbf{1} \sigma_{\varphi} / \mathbf{3} \boldsymbol{\sigma}_{\varphi}\right)\end{array}$} & $\begin{array}{c}\text { PLL loss } \\
\text { lock }\end{array}$ & $\begin{array}{c}\text { Cycle slip rate, } \\
\boldsymbol{P}_{\text {slip }}\end{array}$ \\
\hline D1.1 & 28.5 & 29.7 & 25.9 & 31.2 \\
\hline D1.2 & $\mathbf{2 9 . 8}$ & $\mathbf{3 0 . 9}$ & 25.9 & 28.9 \\
\hline D1.3 & $\mathbf{2 8 . 5}$ & $\mathbf{2 9 . 4}$ & 22 & 21.7 \\
\hline
\end{tabular}

B. D1 types of signal structure candidates

In this section, the candidates signal performances using the structure of the IWG SBAS L5 SIS ICD signal are presented.

TABLE IX. shows the signal performance in $\mathrm{dBHz}$ of:

- D1.1 candidate with a classic interpretation of the cycle slip rate requirement

- D1.2 candidate with alternative interpretation of cycle slip rate requirement. In this case a $P_{\text {slip }}=10^{-3} / \mathrm{s}$ has been chosen which implies a $\mathrm{WER}_{\mathrm{c}}=10^{-5}$ (equation (3))

- D1.3 candidate with a classic interpretation of the cycle slip rate requirement and with additional power used to implement a pilot component.

The performances are presented for a normal $\left(1 \sigma_{\varphi}\right)$ and for the worst $\left(3 \sigma_{\varphi}\right)$ carrier phase estimation error. Carrier tracking is conducted on the data component.

From TABLE IX. , it can be seen that the D1.1 signal threshold (minimum $\mathrm{C} / \mathrm{N}_{0}$ necessary to obtain a correct functioning of the SBAS system) is $31.2 \mathrm{dBHz}$ and is given by the cycle slip rate requirement.

From TABLE IX., it can be seen that the D1.2 signal threshold is between 29.8 and $30.9 \mathrm{dBHz}$ and is given by the message $\mathrm{WER}_{\mathrm{c}}$. Comparing these results with D1.1 (same structure but with traditional cycle slip rate requirement), we can see a decrease of the signal threshold.

From TABLE IX., it can be seen that the D1.3 signal threshold is between 28.5 and $29.4 \mathrm{dBHz}$ and is given by the message $\mathrm{WER}_{\mathrm{c}}$. Therefore, comparing these results with the IWG SBAS L5 SIS ICD signal structure (D1.1), we can see that the introduction of a pilot in addition to providing georanging capabilities, improves the signal threshold by about 2.5 $\mathrm{dB}$.

\section{D2 types of signal structure candidates}

In this section, the candidates signal performances using 1 data component with new LBCCs are presented. Moreover, the increase of data rate, $R_{d}$, is presented.

\section{B.1. Increase of data rate and synchronization component structure}

The increase of the data rate, $R_{d}$, will depend on the type of LBCC being used. Moreover, the $R_{d}$ increase could be further maximized by transmitting the message ID in the synchronization component and by associating to a determined message a type of LBCC.
Therefore, we propose to transmit 7 information bits with the synchronization component:

- $\quad 1$ bit $=$ Determines if the 1 -second length signal block corresponds to the first block of a LBCC

- 6 bits = Message ID

The association between the message ID and the LBCCs will depend on the final signal design. TABLE X. summarizes a possible association made from the fields of the IWG SBAS L5 SIS ICD definition.

TABLE X. ASSOCIATOIN BETWEEN LBCCS AND MESSAGE IDS

\begin{tabular}{|c|c|c|}
\hline CCSDS-250 & CCSDS-500 & CCSDS-1250 \\
\hline $\begin{array}{c}\text { Integrity, Almanac, } \\
\text { Degradations Parameters }\end{array}$ & $\begin{array}{c}\text { Almanac, Degradations } \\
\text { parameters, Ephemeris }\end{array}$ & SV corrections \\
\hline
\end{tabular}

Finally, the $R_{d}$ increase is obtained from the reduction of header bits (synchronization bits, message ID) and CRC bits w.r.t the data bits. Therefore, the final gain in bits per codeword is:

$$
\text { Gain }=(8+6) \cdot S+24 \cdot(S-1)-4^{*}
$$

- $\quad S$ is the number of 1 -second length blocks occupied by the LBCC

- $\quad 4^{*}$ is the number of tail bits required by the selected CCSDS turbo codes. However, if a LDPC channel code was selected, these bits would not be necessary.

TABLE XI. summarizes the gain in bits. From TABLE XI, we can already identify some uses for the available bits: for example, for the integrity message, 10 more bits (or 14 bits for LDPC channel code) are available which allows the inclusion of 2 (or 3) additional DFREI (and thus up to 9 or 10 exhaustively monitored satellites) and 1 additional DFRECI. Moreover, the additional 166 bits which could be used with the CCSDS-1250 could allow the transmission of SV corrections in less time and thus could open the door for Ionospheric corrections and alarms for L5-only transmissions.

\section{B.2. Performances}

TABLE XII. shows the signal performance in $\mathrm{dBHz}$ of:

- D2.1 candidate with a classic interpretation of the cycle slip rate requirement. Carrier tracking is conducted over the pilot component.

- D2.2 candidate with an alternative interpretation of the cycle slip rate requirement: $P_{\text {slip }}=2 \cdot 10^{-4} / \mathrm{s}$ for the longest codeword CCSDS-1250 $(l=5)$ has been chosen which implies a $\mathrm{WER}_{\mathrm{c}}=10^{-5}$ (equation (3)). Carrier tracking is conducted over the data component.

- D2.1 candidate with a classic interpretation of the cycle slip rate requirement and with additional power used to implement a pilot component. Carrier tracking is conducted over the pilot component.

The performances are presented for a normal $\left(1 \sigma_{\varphi}\right)$ and for the worst $\left(3 \sigma_{\varphi}\right)$ carrier phase estimation error and only for the LBCCs code presenting the highest threshold. 
From TABLE XII, it can be seen that D2.1 signal threshold is between 29.2 and $30.9 \mathrm{dBHz}$ and is given by the message WER $_{\mathrm{c}}$. Therefore, comparing these results with D1.1 (TABLE IX. ) performance, we can see that in addition to increasing $R_{d}$, the signal threshold is decreased.

From TABLE XII, it can be seen that D2.2 signal threshold is $30.4 \mathrm{dBHz}$ and is given by the cycle slip rate requirement. Therefore, comparing these results with D1.2 (TABLE IX. ) performance, we can see that the signal threshold is slightly degraded (about $0.5 \mathrm{~dB}$ ) when increasing $R_{d}$.

From TABLE XII, it can be seen that the D2.3 signal threshold is between 27.5 and $28.5 \mathrm{dBHz}$ and is given by the message $\mathrm{WER}_{\mathrm{c}}$. Therefore, comparing these results with D1.3 (TABLE IX. ) performance, we can see that in addition to increasing $R_{d}$, the signal threshold is decreased.

\section{D3 types of signal structure candidates}

In this section, the candidates signal performances using 2 data components, one with the IWG SBAS L5 SIS ICD signal structure and the other with the new LBCCs, are presented. The increase of data rate, $R_{d}$, is given as well. This structure is presented since it will allow a receiver to just process the IWG SBAS L5 SIS ICD signal data component regardless of the other candidate signal components.

\section{C.1. Coordination between data components}

In order to obtain a good performance from the D3 candidates, we need to implement a good coordination between the data components. The most important thing to avoid is the degradation of the integrity/alarm parameters. Therefore, when the integrity/alarm message (and maybe other messages) is transmitted, both data components must transmit the same information.

TABLE XI. GAIN IN BITS WHEN USING LBCCS

\begin{tabular}{|c|c|c|}
\hline CCSDS-250 & CCSDS-500 & CCSDS-1250 \\
\hline 14 & 52 & 166 \\
\hline
\end{tabular}

TABLE XII. D2 CANDIDATES PERFORMANCE SUMMARY. $\mathrm{WER}_{\mathrm{C}}=10^{-3}$ WITH CCSDS-250 FOR BLUE CELLS AND WER C $_{\mathrm{C}}=10^{-5} \mathrm{WITH} \mathrm{CCSDS}^{-1250 \text { FOR }}$ GREEN CELLS. $P_{\text {slip }}=10^{-5} / \mathrm{s}$ FOR PINK CELLS AND $P_{\text {slip }}=2 \cdot 10^{-4} / \mathrm{s}$ FOR VILOET CELLS.

\begin{tabular}{|c|c|c|c|c|}
\hline Candidate & \multicolumn{2}{|c|}{$\begin{array}{l}\text { Secondary PRN code } \\
\text { acquisition, } P_{2 a c q}=10^{-5}, \\
\qquad\left(1 \sigma_{\varphi} / 3 \sigma_{\varphi}\right)\end{array}$} & \multicolumn{2}{|c|}{$\begin{array}{l}\text { Synchronization component } \\
\text { demodulation performance, } \\
\qquad P_{S}=10^{-8},\left(1 \sigma_{\varphi} / 3 \sigma_{\varphi}\right)\end{array}$} \\
\hline D2.1 & 21.8 & 26.2 & 27 & 29.3 \\
\hline D2.2 & 26.4 & 28.1 & 27.7 & 29.1 \\
\hline D2.3 & -- & -- & 24.9 & 26.4 \\
\hline Candidate & \multicolumn{2}{|c|}{$\begin{array}{l}\text { Data component, } W E R_{c} \\
\left(1 \sigma_{\varphi} / 3 \sigma_{\varphi}\right)\end{array}$} & $\begin{array}{l}\text { PLL loss } \\
\text { lock }\end{array}$ & $\begin{array}{l}\text { Cycle slip } \\
\text { rate }\end{array}$ \\
\hline D2.1 & 29.2 & 30.9 & 22 & 26.9 \\
\hline D2.2 & 27 & 28.6 & 26.6 & 30.4 \\
\hline D2.3 & 27.5 & 28.5 & 22 & 21.7 \\
\hline
\end{tabular}

The synchronization component is designed in order to accommodate this need. 8 bits are originally planned but 7 bits will be enough if there is a general guideline specifying which message types can be coherently added:

- (optional) 1 bit = Same information in both components

- 1 bit $=1$-second length signal block of the 2 data component corresponds to the first block of a LBCC.

- (Optional) 6 bits = Message ID (first and/or second component). Each message ID can have a fixed associated LBCC.

\section{C.2. Increase of the bit rate}

The final increase of data rate will depend on the implemented LBCCs of the $2^{\text {nd }}$ data component and on the sensitive information being broadcasted simultaneously on both data components.

For example, if only the integrity information is broadcasted in both data components and using the scheme provided in [3] for 90 satellites, the useful bit information rate is increased by 1.83 times for D3 (and D4) candidates.

Moreover, assuming 2 consecutives integrity messages are sent in order to transmit the DFREI of about 90 satellites, D3 (and D4) candidates will still increase 1.66 times the $R_{d}$ which could be used to transmit Ionospheric corrections and alarms for L5-only (CAT I autoland service provided), degradations parameters per satellites of blocks of satellites instead of constellations, railway or maritime applications related information, ARAIM parameters and GNSS authentication.

\section{C.3. Performances}

TABLE XIII. shows the signal performance in $\mathrm{dBHz}$ of:

- D3.1 candidate with a classic interpretation of the cycle slip rate requirement. Carrier tracking is conducted over the 2 data components.

- No suitable D3.2 candidate, with an alternative cycle slip rate requirement interpretation, has been found.

- D3.3 candidate with a classic interpretation of the cycle slip rate requirement and with additional power used to implement a pilot component. Carrier tracking is conducted over the pilot component.

The performances are presented for a normal $\left(1 \sigma_{\varphi}\right)$ and for the worst $\left(3 \sigma_{\varphi}\right)$ carrier phase estimation error and only for the LBCCs code presenting the highest threshold.

From TABLE XIII, it can be seen that the D3.1 signal threshold is either between 32 and $33.1 \mathrm{dBHz}$ and is given by the message $W R_{c}$ when the IWG SBAS L5 SIS ICD signal data component is decoded alone, or is $32.6 \mathrm{dBHz}$ given by the cycle slip rate. Comparing this value with the D1.1 threshold (TABLE IX. ), we can see that the degradation is about $1.5 \mathrm{~dB}$. This degradation is acceptable with respect to the $R_{d}$ increase.

From TABLE XIII, it can be seen that the D3.3 signal threshold is between 31.9 and $32.5 \mathrm{dBHz}$ and is given by the message $\mathrm{WER}_{\mathrm{c}}$ when the IWG SBAS L5 SIS ICD signal data 
component is decoded alone. Comparing this value with the D1.3 threshold (TABLE IX. ), we can see that the degradation is about $3 \mathrm{~dB}$. However, this degradation is small compared to the D1.1 threshold. Therefore, we can conclude that the inclusion of a pilot component, in addition to provide georanging capabilities, will increase $R_{d}$ with a slight decrease of the original SBAS L1 signal performances.

\section{E. D4 types of signal structure candidates}

In this section, the candidates signal performances using 2 data components implementing new LBCCs are presented. The comparison between D4 and D3 candidates is very interesting since it shows how the 2 data component design is better exploited when LBCCs codes are employed.

The increase of the data rate, $R_{d}$, is the same as for D3 candidates (see section VI.C.2).

\section{D.1. Coordination between data components}

As said for D3 candidates, coordination between the 2 data components is very important to guarantee that sensitive information is always decoded with the lowest threshold.

TABLE XIII. D3 AND D4 CANDIDATES PERFORMANCE SUMMARY. WER $\mathrm{W}_{C}=$

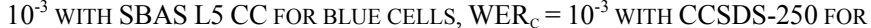
GREEN CELLS, WER $_{C}=10^{-3}$ WITH CCSDS- 1250 FOR PINK CELLS, WER $_{C}=10^{-5}$

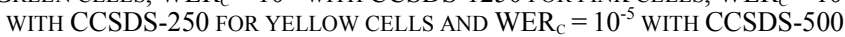
FOR VIOLET CELLS. $P_{\text {slip }}=10^{-5} / \mathrm{s}$ FOR ORANGE CELLS AND $P_{\text {slip }}=10^{-3} / \mathrm{s}$ FOR RED CELLS.

\begin{tabular}{|c|c|c|c|c|}
\hline Candidate & \multicolumn{2}{|c|}{$\begin{array}{c}\text { Secondary PRN code } \\
\text { acquisition, } P_{2 a c q}=10^{-5}, \\
\qquad\left(1 \sigma_{\varphi} / 3 \sigma_{\varphi}\right)\end{array}$} & \multicolumn{2}{|c|}{$\begin{array}{l}\text { Synchronization component } \\
\text { demodulation performance, } \\
\qquad P_{s}=10^{-8},\left(1 \sigma_{\varphi} / 3 \sigma_{\varphi}\right)\end{array}$} \\
\hline D3.1 & 26.6 & 28.5 & 27.8 & 29.4 \\
\hline D3.3/D4.3 & -- & -- & 22.3 & 24.4 \\
\hline D4.1 & 27.2 & 30 & 27.7 & 29.3 \\
\hline$D 4.2$ & 26.4 & 28.4 & 27.7 & 29.3 \\
\hline Candidate & \multicolumn{2}{|c|}{$\begin{array}{c}1^{\text {st }}+2^{\text {nd }} \text { data components, } \\
\text { dem. perfor., } W E R_{c} \\
\left(1 \sigma_{\varphi} / 3 \sigma_{\varphi}\right)\end{array}$} & \multicolumn{2}{|c|}{$\begin{array}{c}1^{\text {st }} \text { data component only, } \\
\text { dem. perfor., } W E R_{c} \\
\left(1 \sigma_{\varphi} / 3 \sigma_{\varphi}\right)\end{array}$} \\
\hline D3.1 & 29.1 & 30.5 & 32 & 33.1 \\
\hline D3.3 & 29 & 29.8 & 31.9 & 32.5 \\
\hline D4.1 & 28.7 & 31 & 31.4 & 33 \\
\hline D4.2 & 28.4 & 29.8 & 31.3 & 32.3 \\
\hline D4.3 & 27.3 & 28.4 & 30.2 & 30.9 \\
\hline Candidate & \multicolumn{2}{|c|}{$\begin{array}{c}2^{\text {nd }} \text { data component only, } \\
\text { demo. Perfor., } W E R_{c} \\
\left(1 \sigma_{\varphi} / 3 \sigma_{\varphi}\right)\end{array}$} & $\begin{array}{l}\text { PLL loss } \\
\text { lock }\end{array}$ & $\begin{array}{l}\text { Cycle slip } \\
\text { rate }\end{array}$ \\
\hline D3.1 & 30.4 & 31.6 & 27.5 & 32.6 \\
\hline D3.3 & 30.2 & 30.9 & 22 & 21.7 \\
\hline D4.1 & 30.5 & 32.3 & 23.7 & 28.7 \\
\hline D4.2 & 30.3 & 31.5 & 27.5 & 30.4 \\
\hline$D 4.3$ & 29.2 & 30 & 22 & 21.7 \\
\hline
\end{tabular}

Additionally, D4 candidates can also broadcast different 1second length blocks of the same codeword in the two data components at the same time. For example, for candidate D4.2, codewords CCSDS-500 which occupies two seconds, can be broadcasted in only 1 seconds if the first 500 coded bits are broadcasted in the $1^{\text {st }}$ data component and the last 500 coded bits are broadcasted in the $2^{\text {nd }}$ data component (see Fig. 10). In this case, the relationship between the cycle slip rate and the codeword WER is equal to (derived from equation (3)):

$$
W E R_{T}=10^{-3}=1-\left(1-P_{\text {slip }}\right)^{l / c}\left(1-P_{w}\right)
$$

- $\quad c$ is the number of data components broadcasting different parts of the same codeword

For D4 candidates, the synchronization component is designed equivalent as for D3 candidates (see section VI.C.1).

\section{D.2. Performances}

TABLE XIII shows the signal performance in $\mathrm{dBHz}$ of:

- D4.1 candidate with a classic interpretation of the cycle slip rate requirement. Carrier tracking is conducted over the pilot component.

- D4.2 candidate with an alternative interpretation of the cycle slip rate requirement: $P_{\text {slip }}=10^{-3} / \mathrm{s}$ for the codeword CCSDS-500 $(l=2, c=2)$ has been chosen which implies a $\mathrm{WER}_{\mathrm{c}}=10^{-5}$ (equation (26)). Carrier tracking is conducted over the 2 data components.

- D4.3 candidate with a classic interpretation of the cycle slip rate requirement and with additional power used to implement a pilot component. Carrier tracking is conducted over the pilot component.

The performances are presented for a normal $\left(1 \sigma_{\varphi}\right)$ and for the worst $\left(3 \sigma_{\varphi}\right)$ carrier phase estimation error and only for the LBCCs code presenting the highest threshold.

From TABLE XIII, it can be seen that D4.1 signal threshold is between 31.4 and $33 \mathrm{dBHz}$ and is given by the message $\mathrm{WER}_{\mathrm{c}}$ when the $1^{\text {st }}$ data component is decoded alone. Comparing this value with the D1.1 signal threshold (TABLE IX. ), we can see that for an average case there is a slight degradation of about $0.5 \mathrm{~dB}$.

Therefore, we can conclude that candidate D4.1 is very interesting for two reasons:

1) Receivers using only the $1^{\text {st }}$ data component case: $R_{d}$ increase defined in section VI.B.1 for a slight decrease of performance.

2) Receivers using the 2 data components case: very significant $R_{d}$ increase defined in section VI.C.2 and the same or even improved performance as IWG SBAS L5 SIS ICD signal.

From TABLE XIII, it can be seen that the D4.2 signal threshold is between 31.3 and $32.3 \mathrm{dBHz}$ and is given by the message $\mathrm{WER}_{\mathrm{c}}$ when the $1^{\text {st }}$ data component is decoded alone. Comparing this value with the D1.1 signal threshold (TABLE IX. ), we can see that for an average case there is a slight degradation of about $1.5 \mathrm{~dB}$. We can conclude: 
1) Receivers using only the $1^{\text {st }}$ data component case: $R_{d}$ increase defined in section VI.B.1 for a $1.5 \mathrm{~dB}$ threshold decrease.

2) Receivers using the 2 data components case: very significant $R_{d}$ increase defined in section VI.C.2 and slight decrease of performance compared to IWG SBAS L5 SIS ICD signal (D1.2 candidate). However, slight better performances than D4.1.

From TABLE XIII, it can be seen that the D4.3 signal threshold is between 30.2 and $30.9 \mathrm{dBHz}$ and is given by the message $\mathrm{WER}_{\mathrm{c}}$ when the $1^{\text {st }}$ data component is decoded alone. Comparing this value with the D1.3 signal threshold (TABLE IX. ), we can see that for an average case there is a slight degradation of about $1.5 \mathrm{~dB}$. However, if both data components are used at the same time the degradation is reduced to $0.5 \mathrm{~dB}$ for a significant $R_{d}$ increase. Moreover, the demodulation performance using 1 or two data components are equal or better than IWG SBAS L5 SIS ICD signal (D1.1 and D1.2 candidates), and we provide improved geo-ranging capabilities.

\section{$F$. Bit rate increase using $C S K$}

The use of a CSK modulation in order to increase the bit information rate is only considered for 2 data components candidates (D3 and D4) and only on the second data component. The reason is that a receiver should always have the possibility of obtaining the information from the IWG SBAS L5 SIS ICD signal structure (or a signal structure which only includes LBCCs) without the need of demodulating a CSK modulation. Moreover, when using a dynamic CSK modulation, the receiver could selectively ignore the 1 -second length signal blocks which are CSK modulated (identified using the synchronization component)

The second data component could implement a static $\mathrm{CSK}(4,10)$ or even more interesting, the dynamic BPSK/CSK modulation presented in section IV.A.2.

The dynamic CSK demodulation performances when using classical CSK decoding are given in TABLE XIV.

TABLE XIV. CSK DEMODULATION PERFORMANCES

\begin{tabular}{|c|c|c|c|c|}
\hline \multirow{2}{*}{$\begin{array}{c}\text { Candidates } \\
\text { D4.1 }\end{array}$} & \multicolumn{2}{|c|}{$\begin{array}{c}\text { CSK }(4,10)+\text { Subframe } 2 \\
\text { GPS L1C LDPC, WER }=10^{-3} \\
\left(\times 2.5 R_{b} \text { of data component) }\right. \\
\left(1 \sigma_{\varphi} / 3 \sigma_{\varphi}\right)\end{array}$} & \multicolumn{2}{|c|}{$\begin{array}{c}\text { CSK }(2,10)+\text { Subframe } 2 \\
\text { GPS L1C LDPC, WER } \text { R }_{c}=10^{-3} \\
\left(\times 5 R_{b} \text { of data component) }\right. \\
\left(1 \sigma_{\varphi} / 3 \sigma_{\varphi}\right)\end{array}$} \\
\hline & 35.4 & 36.3 & 38.3 & 38.9 \\
\hline D3.1/D4.2 & 34.5 & 34.9 & 37.4 & 37.8 \\
\hline D3.3/D4.3 & 34.15 & 34.6 & 37.2 & 37.4 \\
\hline
\end{tabular}

From TABLE XIV., it can be observed that large values of $\mathrm{C} / \mathrm{N}_{0}$ are required to demodulate the targeted CSK modulation. However, the utilization of the CSK modulation should only be thought for non-core services and only for users in good receptions conditions.

\section{TARGET APPLICATIONS/OPERATIONS IDENTIFICATION}

In this section, an identification of the applications which could be targeted by the analyzed signal candidates is presented.
- D2 candidates: Cat I operation with enhanced positioning services (2 or 3 additional DRFEIs for integrity message, degradation parameters for satellite blocks instead of constellations, bias terms of residual errors after application of SBAS SV corrections, Information on SV signal distortions), or even Cat I autoland operation.

- $\quad$ D3 and D4 candidates with/without CSK: Cat I, Cat I autoland and Cat II operations, non-airborne applications (railway and/or maritime), ARAIM parameters and GNSS authentication.

\section{CONCLUSIONS}

In this work, we have analyzed several signal design candidates which pursue 3 main goals. The first goal is to increase the signal data rate $\left(R_{d}\right)$ with respect to IWG SBAS L5 SIS ICD in order to enhance aviation positioning services, in order to provide non-aviation services (maritime, railway) and in order to provide additional information for additional services such as ARAIM parameters and GNSS authentication. The second goal is to keep a $100 \%$ compatibility with the IWG SBAS L5 SIS ICD definition (possibility of alarm every integer number of seconds, etc). The third and last goal consists in preserving the IWG SBAS L5 SIS ICD signal performance.

The analyzed signal candidates presented as major improvements:

- Implementation of powerful linear block channel codes (LBCC) in order to reduce the demodulation threshold and in order to decrease the header/CRC bits w.r.t. the data bits.

- Introduction of a synchronization component in order to synchronize and to identify the different LBCCs.

- Introduction of a pilot component in order to synchronize LBCCs, in order to decrease the cycle slip rate threshold and in order to provide enhanced georanging capabilities (optional).

- Introduction of a second data component in order to increase the signal data rate, $R_{d}$.

- And implementation of a CSK modulation in order to increase the signal data rate, $R_{d}$.

All the signal design candidates present a backward compatibility with the IWG SBAS L5 SIS ICD PRN code signal structure. The only exceptions are candidates selectively having 1-second length blocks of their second data component that are CSK modulated. However, a receiver can choose to ignore the CSK modulated 1-second length signal blocks (identified using the synchronization component) or to completely ignore the $2^{\text {nd }}$ data component. Therefore, the backward PRN code structure compatibility is guaranteed.

The signal design candidates implementing 1 data component and new LBCCs had about the same or better demodulation performance than the IWG SBAS L5 SIS ICD signal. Moreover, they obtained a significant reduction of header/CRC bits w.r.t to the data bits. 
The signal candidates with 2 data components were designed to allow the receiver to select the number of data components which he wanted to process. Therefore, a receiver had the possibility to process just one data component which provides the IWG SBAS L5 SIS ICD signal (implementing CC or LBCCs) without concerns for the second data component. Processing both data components allows the user to obtain enhanced aviation positioning services w.r.t the services provided by the current IWG SBAS L5 SIS ICD signal, and it also allows the user to obtain non-aviation services (railway, maritime) and other services (GNSS authentication, etc).

Two types of signal design candidates with 2 data components were presented. The first type implemented the IWG SBAS L5 SIS ICD signal in the $1^{\text {st }}$ data component and the second type implemented LBCCs on both data components. The second type of candidates provided better performance than the first type since LBCCs allow a better exploitation of the 2 data components: the performances are almost equivalent to the IWG SBAS L5 SIS ICD signal but with a very significant $R_{d}$ increase: an increase of at least 1.66 times the original $R_{d}$. Moreover, the inclusion of a CSK modulation on the second data component allowed an even further increase of $R_{d}$ but at the cost of a higher $\mathrm{C} / \mathrm{N}_{0}$ threshold for these specific parts.

Finally, the introduction of a pilot component with additional allocated power to provide enhanced geo-ranging capabilities improved the demodulation performance of the signal design candidates and the IWG SBAS L5 SIS ICD signal. In fact, for the 2 data components signal candidates implementing LBCCs, the introduction of a pilot component with additional power allows to enhance IWG SBAS L5 SIS ICD geo-ranging capabilities, to significantly increase the signal data rate $R_{d}$ (at least 1.66 times) and even to improve the IWG SBAS L5 SIS ICD signal threshold.

\section{ACKNOWLEDGMENT}

This work was developed on the framework of a research project financed by CNES and was under the management of Egis Avia.

\section{REFERENCES}

[1] P. Enge and A.J. Van Dierendonck, "Design of the Signal and Data Format for Wide Area Augmentation of the Global Positioning System", ION PLANS 1996, Atlanta GA, 22-26 Apr 1996.

[2] P. Enge, "WAAS Messaging System: Data Rate, Capacity, and Forward Error Correction", NAVIGATION, Journal of The Institute of Navigation, Vol. 44, No. 1, Spring 1997, pp. 63-76.

[3] T. Walter, J. Blanch and P. Enge, "L1/L5 SBAS MOPS to Support Multiple Constellations", ION GNSS+ 2012, Nashville TN, September $17-21,2012$

[4] T. Walter, J. Blanch and P. Enge, "Implementation of the SBAS L5 Mops”, ION GNSS+ 2013, Nashville TN, September 16 - 20, 2013
[5] M. Cueto et al., SBAS L1/L5 ICDs and Alternatives: Analysis of MultiGNSS Service Performance Assessment, ION GNSS+ 2013, Nashville TN, September 16 - 20, 2013

[6] EGNOS V3 MRD V1.0 (internal EC document)

[7] RTCA DO 229D, Minimum Operations Performance Standards for Global Positioning System/Wide Area Augmentation System Airbone Equipment.

[8] RTCA DO 235B, Assesment of Radio Frequency Intereference Relevant to the GNSS L1 Frequency Band to the GNSS Frequency Band.

[9] C. Hegarthy, "Analytical Derivation of Maximum Tolerable In-Band Interference Levels for Aviation Applications of GNSS", Proceedings of the 9th International Technical Meeting of the Satellite Division of The Institute of Navigation, ION GPS 1996, September 17 - 20, 1996, Kansas City, MO.

[10] M. Tran, C. Hegarthy and A.J. Van Dierendonck, "SBAS L1/L5 Signal Design Options", Proceedings of the 59th Annual Meeting of The Institute of Navigation and CIGTF 22nd Guidance Test Symposium, June 23 - 25, 2003, Albuquerque, NM

[11] EU-US Cooperation on Satellite Navigation, Working Group C, Combined Performances for SBAS Receivers for SBAS using WAAS and EGNOS, July 19, 2010.

[12] ARINC Engineering Services, "Navstar GPS space Segment/User segment L5 interfaces, Draft IS-GPS-705”, Sept 22, 2005

[13] ARINC Engineering Services, "Navstar GPS space Segment/User segment L1C interfaces, IS-GPS-800A", June 08, 2010

[14] European Space Agency, "Galileo OS SIS ICD Issue 1.1", September 2010

[15] A. Garcia-Pena, D. Salos, O. Julien, L. Ries and T. Grelier, "Analysis of the use of CSK for Future GNSS Signals", Proceedings of the 26th International Technical Meeting of The Satellite Division of the Institute of Navigation (ION GNSS+ 2013), September 16 - 20, 2013, Nashville, TN

[16] M. Anghileri, M. Luise, A. Perotti, A. Tarable, M. Paonni, B. Eissfeller, G. Lopez-Risuenõ and F. Zanier, "A fresh look into designing channel error protection codes for satellite navigation messages", $5^{\text {th }}$ European Workshop on GNSS Signals and Signal Processing, 2011, Toulouse.

[17] J.G Proakis and M.Salehi, "Digital Communications" 5th ed, McGrawHill, 2008.

[18] Consultative Committee for Space Data Systems (CCSDS), "Blue Book: TM Synchronization and channel coding", August 2011.

[19] S. Dolinar and D. Divsalar, "Weigth distribution of TurboCodes using Random and Non-random permutations", TDA progress report 42-122, Jet Propulsion Laboratory, August 1995

[20] E. Kaplan and C. Hegarty, "Understanding GPS: Principles And Applications", Artech House, 2005.

[21] O. Julien, J-L. Issler and L. Ries, "Investigation of Galileo E1 OS/SoL Acquisition, Tracking and Data Demodulation Thresholds for Civil Aviation", Proceedings of the 24th International Technical Meeting of The Satellite Division of the Institute of Navigation (ION GNSS 2011), September 20 - 23, 2011, Portland, OR

[22] S.A.Stephens and J. B. Thomas, "Controlled-Root Formulation for Digital Phase-Locked Loops", IEEE Transactions on Aerospace and Electronic Systems, 1995 